\title{
Septins disruption controls tumor growth and enhances efficacy of Herceptin
}

Rakesh K Singh*1, Kyu Kwang Kim¹, Negar Khazan, Rachael B. Rowswell-Turner ${ }^{1}$, Christian Laggner $^{3}$, Aaron Jones ${ }^{1}$, Priyanka Srivastava ${ }^{1}$, Virginia Hovanesian ${ }^{4}$, Liz Lamere ${ }^{1}$, Thomas Conley ${ }^{1}$, Ravina Pandita ${ }^{1}$, Cameron Baker ${ }^{5}$, Jason R Myers ${ }^{5}$, Elizabeth Pritchett ${ }^{5}$, Awada Ahmad $^{1}$, Luis Ruffolo ${ }^{2}$, Katherine Jackson ${ }^{2}$, Scott A. Gerber², John Ashton ${ }^{5}$, Michael T. Milano ${ }^{6}$, David Linehan $^{2}$, Richard G Moore ${ }^{1}$

${ }^{1}$ Wilmot Cancer Institute, Division of Gynecologic Oncology, Department of Obstetrics and Gynecology, University of Rochester Medical Center, Rochester, NY, USA.

2Department of Surgery, Microbiology and Immunology; Department of Radiation Oncology and Center for Tumor Immunology Research, University of Rochester Medical Center, Rochester, NY, USA.

${ }^{3}$ Atomwise Inc, San Francisco, CA, USA.

${ }^{4}$ Rhode Island Hospital, Providence, RI, USA.

${ }^{5}$ Genomics Research Center, Wilmot Cancer Center, University of Rochester Medical Center, NY, USA.

${ }^{6}$ Department of Radiation Oncology, University of Rochester, NY, USA.

"Corresponding author:

Rakesh Singh@URMC.Rochester.Edu

Telephone (office): 585-276-6281. Fax: 585-276-2576

\begin{abstract}
Septin expressions are altered in cancer cells and exhibit poor prognoses in malignancies. As the first approach to develop a septin filament targeting agent, we optimized the structure of Forchlorfenuron (FCF), a known plant cytokinin to generate UR214-9, which contrary to FCF, causes septin-2/9 filamental structural catastrophe in cancer cells without altering cellular septin protein levels. In-silico docking using septin-2/septin-2 dimer complex showed that UR214-9 displaced the guanine carbonyl oxygen from the GDP binding domain and showed increased binding energy than FCF(-8.59vs-7.21). UR214-9 reduced cancer cell growth, downregulated HER2/STAT-3 axis and controlled growth of HER2+ pancreatic, breast and ovarian cancer xenografts in NSG mice and enhanced response of Herceptin against HER2+breast cancer xenograft. Transcriptome analysis of UR214-9 exposed cells demonstrated significant perturbation of $<20$ genes compared to afatinib which impacted $>1200$ genes in JIMT- 1 breast cancer cells indicating target specificity and non-transcriptional functions of UR214-9. In summary, disrupting septins via UR214-9 is a new approach to control the growth of HER2+ malignancies.
\end{abstract}

\section{Introduction}

Septins are a family of GTP-binding cytoskeletal proteins that participate in cytokinesis, cell migration, chromosomal dynamics and protein secretion. Septins hetero-oligomerize to generate scaffolding filaments, bundles, and rings within cells $\mathbf{s}^{1-11}$. Additionally, septins are a critical cytoskeletal component that regulate the function of tubulin and actin. Altered septin protein expression in pancreatic, kidney, lung, colorectal, skin, brain, endometrial, ovarian, breast and other malignancies have been observed ${ }^{12-16}$. Aberrant septin expression has also been linked to neurodegenerative/neuromuscular diseases, blood disorders, infertility, and developmental disabilities ${ }^{17-19}$. It is unclear whether aberrant enrichment of individual septin family members is enough to enhance tumorigenesis or if a specific hetero-oligomer assembly may be implicated. 
Pharmacologic agents to target septins have remained elusive, largely because the oligomeric structural configurations of septins pose difficult challenges in designing therapies.

In this study, we investigated the impact of individual septins on the survival of patients with cancers of the pancreas, breast, lung, kidney, or liver cancer or with melanoma. To determine the effect of septins on survival, we used the Human Protein Atlas (HPA), and publically available transcriptional data and tools available at R2:Genomics Analysis and Visualization Platform ${ }^{20}$ (https://hgserver1.amc.nl/cgi-bin/r2/main.cgi). We describe a potent septin modulator, UR214-9, which disrupts structural organization of septin- 2 and septin- 9 as well as of $\beta$-actin, and controls cancer cell proliferation and tumor growth. We have employed molecular docking techniques to investigate how UR214-9 and its analogs interact with the elements of the GDP binding domain, and of the known FCF binding pocket. To identify how gene expression is impacted by UR214-9, and thereby characterize its off-target liabilities, we have conducted transcriptome analyses of UR214-9 treated breast and pancreatic cancer cells. In summary, our studies present UR214-9, as a potent and novel septin filamental modulator and demonstrate that the dismantling of septin structures in pancreatic, ovarian and breast cancer cells by UR214-9 can be an effective therapeutic strategy.

\section{Results}

\section{Enrichment of septins correlates with decreased survival in patients with cancer}

Publically accessible microarray data bases of pancreatic cancer and ovarian cancer patients deposited at R2:Genomics Analysis and Visualization Platform(https://hgserver1.amc.nl/cgibin/r2/main.cgi) were analyzed. Septin-2 mRNA was enriched in malignant pancreas compared to normal pancreas (Figure-1A, $p=1.3 e^{-4}$ ). Similarly, ovarian cancer epithelium expressed significantly enrichment of septin-2 compared to normal stroma (Figure-1B-left, $p=1.2 e^{-7}$ ). Microdissected stroma of malignant ovarian stroma was also exhibited elevated expression of septin2 mRNA than normal stroma (Figure-1B-right, $p=1.21 \mathrm{e}^{-4}$ ). Similarly, compared to normal stroma, tumor epithelium components of malignant breasts showed increased septin-2 mRNA enrichment (Figure-1C-left, $p=0.49 \mathrm{e}^{-3}$ ). Further, increase in invasive area of breast tumors led to increased septin-2 enrichment (Figure-1C-right, $p=8.9 e^{-3}$ ). Kaplan-Meier survival of patients with pancreatic cancer, grouped by the extent of septin-2 expression (from microarray data available at https://hgserver1.amc.nl/cgi-bin/r2/main.cgi ${ }^{20}$ and Human Protein Atlas ${ }^{21}$, show that septin-2 mRNA enrichment significantly $(p=0.0011)$ correlates with increased mortality (Figure 1D-left). Similarly, enrichment of septin-7 and -9 correlates with increased mortalities in pancreatic cancer patients (Supplementary Figure 1). Septin-2 enrichment is also an unfavorable factor for patients with breast (Figure-1D, middle, $p=3.9 e-3$ ) and ovarian cancer (Figure-1D, right, $p=0.011$ ). Analysis of the survival prospects based on other septins indicate that septin-7 enrichment was found to be unfavorable for the patients diagnosed with malignancies of breast $(p=0.0079)$ (Supplementary Figure-1).

\section{UR214-9 causes septin-2 catastrophe in cells}

The chemical structure of UR214-9 is shown in Figure-2A. UR214-9 was obtained by the structure-activity relationship guided optimization of FCF. Incorporation of a group of fluorine atoms on phenyl ring and installation of a chlorine atom at C-6 of pyridine ring made UR214-9 a potent disruptor of septin's filamental structure. Confocal microscopy at higher resolution $(60 \times 2)$ was employed to determine the impact of DMSO, FCF (+ve control) and UR214-9 on the structural arrangement of septin-2, 6, 7 and -9 in a panel of BXPC-3, CAPAN-1, Panc-1 (pancreatic) and JIMT-1 (breast) and SKOV-3 ovarian cancer cells. While FCF seems to strengthen the septin-2 filaments in BXPC-3 cells (Figure-2B, left-lower), Septin-2 needles in PANC-1 were disarranged 
and translocated at the cell-surface after UR214-9 treatment (1uM) (Figure 2C, lower left). Similarly, the septin-2 needles in JIMT-1 cells after drug treatment showed structural disruptions and relocation to nuclear periphery (Figure-2C, right-lower). Next, the confocal microscopy was employed to investigate the response of other septin family members in PANC-1 cells upon treatment with UR214-9. Septin-7 showed reduced expression whereas septin-9 showed disarrangement of filamental structure (Supplementary Figure-2). Septin-4, -6, did not exhibit clear filamental structures and showed punctate staining instead, which was either reduced in the treatment group compared to DMSO treated control or the drug effect was inconclusive (data not shown). Similarly, UR214-9 treated JIMT-1 breast cancer cells showed strong structural disarrangement and relocation of septin-2 on the periphery of nucleus (Figure-2, right). JIMT-1 cells did not exhibit defined septin-7 structures, and therefore, the effect of UR214-9 on septin-7 remains ambiguous (Supplementary Figure-2, lower left). However, the confocal microscopy of PANC-1 and JIMT-1 cells treated with UR214-9 exhibited clear disarrangement in septin-9 filament structures (Supplementary Figure-2, lower right). Whether UR214-9 treatment alters expression of septin family of proteins was investigated by immunoblotting the total cell-lysates of PANC-1, MDA-MB-231, JIMT-1 and MCF-7 cancer cells. The immunoblots were probed with validated septin-2, 6,7 and -9 antibodies. In PANC-1 cells, septin-9 expression was completely inhibited intriguingly, while expression of septin-2, -6 and -7 were unaffected (Figure-2D). Similarly, western blot analysis of MDA-MB-231, JIMT-1 and MCF-7 cells showed that UR214-9 does not alter the protein expression levels of septin-2,6 and -9 family of proteins (Figure-2E) even though their filamental structures are overwhelmingly disrupted. Septin catastrophe phenomenon in cancer cells was further validated using SKOV-3 ovarian cancer cells that upon treatment with UR214-9 $(1 \mu \mathrm{M}, 48$ hours) showed complete disruption of septin-2 filaments wherein septin-2 appears to have relocated to cell surface after drug exposure (Figure-2F). Further examination of septin-6, 7 and -9 structures in drug treated SKOV-3 cells showed reorganization of septin-9 (Figure-2G, lower). Septin-6 was found to be non-needle-like and decreased upon treatment with UR214-9 (Figure-2G, upper). Changes in septin-7 expression were not clear due to non-needlelike and diffused/punctated expression of UR214-9 (Figure-2G, middle).

UR214-9 causes actin filamental disruption in pancreatic and breast cancer cells. Septins have been previously shown to control the function of actin ${ }^{22}$. Confocal microscopy of UR214-9 treated PANC-1 and JIMT1 cells exhibited actin filament disruption (Figure-3) when treated at a dose of $1 \mu \mathrm{M}$ for 48 hours. Representative structural disarrangement of actin filamental needles, for both PANC-1 and JIMT1 cells, Figure-3. Area of interest are shown in shown in the white boxes.

\section{In Silico docking shows key interactions of UR214-9 with septin-2}

Docking experiments to investigate the potential binding mode of UR214-9 and related compounds (including FCF) were performed. UR214-9 and its analogs are smaller in size and similar in structure and symmetry; they consist of a central urea group flanked by two lipophilic substituted aromatic rings. Compounds UR214-8, 9, and 10 are the most active compounds that we have developed; taking this into account, we hypothesized that they could share a similar binding mode (the structure of compounds UR214-8, 9 and 10 are shown in Supplementary Figure 3). Thus, compounds FCF, UR214-8, UR214-9, and UR214-10 were docked into the nucleotide binding site of PDB ID 2QNR, which is the highest quality structure of a septin-2 dimer complex available ${ }^{23}$. Upon visual inspection of the docking poses, two sets of low energy poses ("set A, upper and lower" and "set B, upper and lower") were identified in which all highly active compounds are able to adopt similar conformations. The two sets are similar to each other in that the three main portions of the molecules - the central urea moiety, the pyridine and the phenyl ring - are in roughly the same area, with the pyridine ring taking the place of the guanine in GDP (Figure 4). In set $A$, the pyridine nitrogen atom is seen taking the place of the guanine carbonyl 
oxygen atom, making a hydrogen bond with the backbone of G241 of chain A. ICM scores of setA were found to be compound UR214-8:-8.85, compound UR214-9: -8.59, compound UR214-10: -10.4 and FCF: -7.21 indicating stronger binding energy of the synthesized analogs than the parent FCF. Set B appears to be identical to a previously reported docking pose for FCF in the same structure template, obtained with the Autodock software ${ }^{23}$. In Figure-4C and D, the identity of amino acid residues interacting with atoms of UR214-9 or its analogs are shown.

\section{UR214-9 impairs cancer cell viability and blocks cell cycle progression}

Treatment with UR214-9 reduced the viability of human pancreatic cancer cells (BXPC-3 and PANC-1) cells (Figure 4A) during 72 hours of treatment. PANC-1 and BXPC-3 cells upon treatment with UR214-9 exhibited a large population of non-viable cells based upon staining by the Live-Dead cell kit and by flow cytometry following 72 hours of treatment (Figure 4B and -4C). Given the role of septins in the cell cycle process, we analyzed the effect of UR214-9 on cell cycle progression of PANC-1 and BXPC-3 pancreatic cancer cells at a non-cytotoxic concentration of 100nM. Treatment with UR214-9 at 100nM dose caused minor S-phase arrest in PANC-1 while BXPC-3 cells showed no change in cell cycle distribution at the non-toxic doses (Supplementary Figure-2A). Increasing the dose to $3 \mu \mathrm{M}$ concentration of UR214-9 caused overwhelming arrest in G1 phase ( 95\% compared to $21 \%$ ) of BXPC-3 cells, while PANC-1 cells showed complete arrest in sub-G1/G0 phase (data not shown). Similarly, JIMT-1 cells treated with an increased dose $(3 \mu \mathrm{M})$ of UR214-9 exhibited G1 phase arrest and showed largely increased accumulation in G0-phase (Supplementary Figure-2B)

\section{Analysis of cell cycle protein expression}

The spotted antibody array was employed to simultaneous study multiple cell-cycle related proteins expressed in drug treated or naïve PANC-1 cells. Measurement of relative photon counts showed that Cullin-3, glycogen synthase kinase-3 (GSK-3b), p19ARF, 14.3.3.Pan, APC11, APC2, ATM, C-able, CD14Aphsophatase, CDC25C, CDC34, CDC37, CDC47, CDC7, CDH1, CDK1 and CDK-3 were the most expressed and affected proteins in the treated vs naïve PANC-1 considering $\geq 2.0$ fold change as meaningful (Supplementary Figure-3C). $\beta$-actin showed the most pronounced expression but expression levels remained unchanged after treatment. On the other hand, Cullin-3 showed most pronounced upregulation in the treated versus naïve PANC-1 cells (Supplementary Figure-3, upper). Cullin-3, a member of the cullin-based ubiquitin ligase family interacts with Hrt1 and BTB domain containing proteins. The resulting complex functions as a Cullin3-based E3 ligase to bring specific substrates to ubiquitinylation and degradation ${ }^{24}$ indicating the role of septins in suppression of cullin-3 mediated ubiquitinylation and subsequent degradation.

\section{UR214-9 treatment slows the growth of HER2 +xenograft tumors}

Septin-2 regulates HER2 expression in gastric cancer cells ${ }^{25}$. HER2 is over-expressed in diverse variety of malignancies ${ }^{25-27}$ and is known to promote tumor development, progression, metastasis and chemoresistance ${ }^{28}$. Septins are shown to protect and stabilize HER2 receptor at the plasma membrane of tumor cells to perpetuate the HER2 orchestrated tumorigenesis ${ }^{29}$. We postulated that targeting septin-2 can potentially emerge as a novel approach to control HER2 orchestrated tumorigenesis. A MTS assay showed that UR214-9 treatment reduced the growth of BXPC-3 and PANC-1 pancreatic cancer dose dependently by $48^{\text {th }}$ hour of drug exposure (Figure-5A). Treatment with UR214-9[3 $\mu \mathrm{M}]$ created $29.4 \%$ dead cells during 48 hours of drug exposure when the total population of BXPC-3 cells was analyzed by Live-dead kit (Invitrogen Inc). Similarly, 


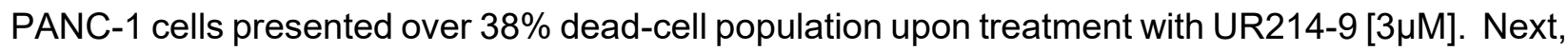
we determined the impact of UR214-9 treatment on pancreatic cancer xenograft tumor growth in vivo. Mice xenografted with HER2+ PANC-1 cells showed significantly delayed growth $(p \leq 0.0001)$ (Figure -5D). The antitumor efficacy of UR214-9 was further evaluated against HER2 positive xenografts derived from JIMT1 (breast cancer) cells. In addition to increased cell death of JIMT1 cells upon UR214-9 exposure in vitro (Figure-5E), JIMT1 xenograft tumors treated with UR2149 casted a significant growth control (Figure-5F), based on both tumor volume and weight measurements (Figure-5G).

\section{UR214-9 inhibits HER2 expression and blocks phosphorylation of STAT-3}

Immunoblot analysis of the total cell lysates of pancreatic cancer cells PANC-1 (HER2+), cells treated with UR214-9 for 72 hours showed a dose-dependent decrease in HER2 expression in PANC-1 (Figure 6A, upper). STAT3 phosphorylation is a down-stream readout of HER2 activation $^{33}$, accordingly UR214-9 treatment also reduced phosphorylated STAT-3 in PANC-1 (Figure 6A, lower) cells. Similarly, UR214-9 treatment reduced HER2 expression in a panel of MDA-MB-231, JIMT-1 and MCF-7 breast cancer cells (Figure-6B) and reduced phosphorylation of STAT-3 in each cell-lines (Figure-6C). We have recently shown that septin-2 is highly overexpressed in ovarian cancer ${ }^{30}$. Similar to JIMT-1 and PANC-1 cell-lines, SKOV-3, a platinum resistant ovarian cancer cell-lines is characterized by HER2 amplification ${ }^{31-32}$. We, therefore employed SKOV-3 cell-line derived xenografts to validate the antitumor efficacy of UR214-9 against HER2 amplified xenograft tumors. To further ascertain the outcome of the combination of UR214-9 with Herceptin, mice were additionally treated with Herceptin alone or in combination with UR214-9. As shown in the Figure-6D, both UR214-9 and Herceptin controlled the growth of tumors. The combination clearly, controlled the tumor growth to a greater degree than both the drugs alone. The real benefit of combination of UR214-9 with Herceptin became apparent when treatments were stopped and tumors were allowed to grow. As shown in the Figure-6D while tumor sizes in UR214-9 and Herceptin group reached the average size in control when the treatments were stopped, the combination maintained greater control over tumor growth (combination $p<0.0001^{* * * *}$ vs $p=0.0004^{* * *}$ and $0.0001^{* * *}$ for vehicle vs UR214-9 and Herceptin). When extracted tumors were weighed, the combination group exhibited presence of smaller tumors, whereas, both the UR214-9 and Herceptin group produced the tumors that matched the average size seen in vehicle group (Figure-6E).

\section{Whole transcriptome analysis reveals that UR214-9 is target selective}

RNA-Seq was performed in the JIMT-1 and Panc-1 cell lines with three treatment groups (10nM Afatinib, $1 \mu$ M UR214-9, and DMSO) of four replicates each. The samples were sequenced to an average depth of 58 million reads and greater than $90 \%$ of the read data for each sample aligned uniquely to the human reference genome (hg38) after adapter and quality trimming. The drug treatments were compared to the control group and differentially expressed genes were determined (adjusted p-value < 0.05). There were 1236 (713 up and 523 down) dysregulated genes between Afatinib treatment and control (Figure 7A and 7C). The ENRICHR webtool was used to determine that the upregulated genes (ALPP, TRIM29, CYP1A1) are associated with extracellular matrix organization and cadherin binding, while the down regulated genes (EGR1, DUSP6, HMGA2, etc.) are associated with purine metabolism and ribosome biogenesis. Conversely, only 11 ( 7 up and 4 down) genes were called dysregulated between UR214-9 treatment and control (Figure 7B and 7D). In terms of the PANC-1 cell line, there were only two genes (COL13A1 and PRSS22) determined to be significantly differentially expressed upon Afatinib treatment compared to the control group and no differentially expressed genes was called between UR214-9 and the control.

\section{Methods}




\section{Cell lines, cell culture and reagents}

PANC-1, BXPC-3 and CAPAN-1, SKOV-3, MCF7, MDA-MD-231 cells were obtained from ATCC and maintained in DMEM, RPMI-1640 and IMDM supplemented with $10 \%$ fetal calf serum penicillin $(100$ units $/ \mathrm{mL})$, and streptomycin $(100 \mu \mathrm{g} / \mathrm{mL})$ at $37^{\circ} \mathrm{C}$ with $5 \% \mathrm{CO}_{2}$ in a humidified incubator. JIMT-1 cells were purchased from AddexBio Inc, USA (catalog number: C0006005) and maintained in 10\% FBS and antibiotic supplemented DMEM. Septin-2 (catalog number: HPA018481), septin-7 (catalog number: HPA029524), septin-9 (catalog number: HPA029524) antibodies were purchased from Sigma Aldrich Inc. DyLight 488 (catalog number: DI-1488, rabbit, Dylight594 (catalog number: DI-2594, mouse) were purchased from Vector Laboratories Inc. Phalloidin-TRITC was purchased from ECM Biosciences (catalog number: PF7551). HER2 (Cell Signaling Technology, catalog number: 4290); pSTAT-3 (catalog number: 9145p), STAT-3 (catalog number: 4904) and GAPDH antibodies (catalog number: 2118s) were purchased from Cell Signaling Technology Inc. USA and used at manufacturer recommended dilutions.

\section{Synthesis of FCF analogs}

UR214-9 was synthesized by coupling aryl isocyanates with 2,6-dichloro 4-aminopyridines in (0.1:0.1) molar ratio in dry DMF at $65^{\circ} \mathrm{C}$ overnight under an argon flushed atmosphere. The reaction was monitored using thin-layer chromatography plates with $\mathrm{DCM}-\mathrm{MeOH}$ or pure ethyl acetate as eluent. Spots were monitored in a UV chamber. The reaction mixture, upon completion of the reaction, was poured into wet ice mixture and triturated and the separated solid was filtered under vacuum. The product was washed with hexane, followed by diethyl ether, and was dried under vacuum. The compounds were characterized by mass spectrometry.

\section{Molecular docking}

Docking experiments to investigate the potential binding mode of 9 and related compounds were performed using Molsoft's ICM software package (v. 3.8-7). The molecules are rather small and somewhat symmetric (consisting of a central urea group flanked by two lipophilicly substituted aromatic rings). We assumed that since compounds 8,9 , and 10 are the most active ones, that they might share a similar binding mode. Thus, compounds FCF, UR214-8,- 9, and -10 were docked into the nucleotide binding site of PDB ID 2QNR, which is the highest quality structure of a septin-2 dimer complex available to date ${ }^{23}$. Receptor preparation (based on the GDP binding site in chain A) and ligand construction was performed within ICM using standard settings. ICM scores for each compound and their poses were calculated and compared with FCF. The compounds were docked with the "dock table" functionality, with a setting for effort of 2.0 and 20 poses per compound. Upon visual inspection of the docking poses, two sets of low energy poses ("set A" and "set B") stood out, in which the highly active compounds are able to adopt similar conformations ${ }^{23}$.

\section{Cell Viability and cell cycle analysis,}

Cell viability of PANC-1, BXPC-3 and CAPAN-1 pancreatic cancer cells treated with UR214-9 was measured using the Cell Titre $96^{R}$ Aqueous One Solution Cell Proliferation Assay (Promega Corp., catalog number: G3580) following the procedure published earlier. The Live/Dead dye kit (Invitrogen Coro., catalog number: L34975) was used to estimate live and dead cell population in PANC-1 and BXPC-3 pancreatic cancer cells treated with UR214-9 or vehicle. Briefly, cells were treated with vehicle or UR214-9 $(3 \mu \mathrm{M})$ for 72 hours. Cells were harvested by trypsinization, fixed and permeabilized using Fixation-Permeabilization reagent (prepared by diluting the concentrate in the diluent in the ratio 1: 3) (Biogem Inc., diluent: catalog number 92160-00-160 and concentrate catalog number: 2550-00-50) and stained with Live/dead dye for one hour. The cells were centrifuged at $1000 \mathrm{rpm}$ for 5 minutes and pellets were washed and spun down three times 
with DPBS. The cells were analyzed by a flow cytometer and relative live and dead cell population was calculated by inputing equal number of cells in both vehicle and control group.

For cell cycle analysis, BXPC-3 and PANC-1 and JIMT-1 cells (100,000/well) were seeded overnight in a 6 well dishes and allowed to adhere overnight. Media was replaced with fresh complete medium supplemented with DMSO or UR214-9 (100nM and $3 \mu \mathrm{M})$ and cells were incubated for 72 hours. The media containing the drugs was removed and cells were washed twice with PBS and trypsinized gently. The cells were collected in $15 \mathrm{~mL}$ tubes, complete DMEM media was added to block trypsin and cells were centrifuged. The supernatants were removed and cells were gently treated with $70 \%$ cold-EtOH for 30 minutes. The fixed cells were centrifuged and the pellets obtained were collected in flow cytometry tubes and stained with preformulated $\mathrm{PI} / \mathrm{R}$ Nase solution (Cell Signaling Technology, catalog number: 4087s) for 30 minutes. The PI content was analyzed using a flow cytometer. Data was processed using Flowjo software.

\section{Cell cycle protein expression}

Cell Cycle Antibody Array (FullMoon BioSystems Inc, catalogue number: ACC:058), a highthroughput ELISA based antibody array, designed for qualitative/semi-quantitative protein expression profiling was employed to investigate the protein changes after drug treatment. PANC1 cells were lysed in buffer containing protease and phosphatase inhibitors (Cell Signaling, catalog number: 9803S). Total protein content was quantified by Bradford assay and equal amounts of proteins were analyzed in duplicate with arrays containing 4 to 6 spots for each of 60 probes (ACC058, Cell Cycle Antibody Array; Full Moon Biosystems, Sunnyvale, CA), according to manufacturer's instructions. After background correction, mean signal intensities were measured using FullMoon Inc's imaging services. Protein expressions in both the naïve and treatment group was normalized to GAPDH signals.

\section{Confocal analysis of septin disarrangement}

To determine the impact of UR214-9 treatment on Septin-2 structure in cells, PANC-1 or JIMT-1 cells were seeded on glass slides and allowed to adhere overnight. The media was replaced with complete DMEM media supplemented with DMSO or UR214-9 (1 $\mu$ M and 70nM) and cells were incubated for 48 hours. Media was replaced again with new complete medium and fixed with neutral buffered formalin for 15 minutes at $4^{\circ} \mathrm{C}$. Media was removed and cells were washed repeatedly with PBST $(5 \times 5 \mathrm{~mL})$. The cells were stained with Septin-2 antibody (Sigma Aldrich, catalog number: HPA018481) in PSB overnight at $4^{\circ} \mathrm{C}$. Media was removed again and cells were washed with $2 \times 5 \mathrm{~mL}$ PBST. The cells were stained with fluorescence linked secondary antibody for $1 \mathrm{hr}$ under dark. Slides were washed repeatedly in dark for $7 \times 5 \mathrm{~mL}$ PBST, mounting medium containing DAPI (Vector labs) were applied and covered with glass slide. The slides were stored in dark at $4^{\circ} \mathrm{C}$ till analysis. Confocal images were obtained and processed essentially as published earlier ${ }^{34}$. Pancreatic tumor microarray (US Biomax, cat no: T142a) were deparaffinized, processed and stained with Septin-2 antibody (Sigma Aldrich, cat number: HPA018481) overnight, washed with PBST and incubated with source matched secondary (FITC) for an hour. Slide was washed in PBST $(5 \times 10 \mathrm{~mL})$ for five minutes each. DAPI containing mounting media was applied and covered with a glass slide. Confocal images were acquired with Nikon C1si confocal microscope (Nikon Inc. Mellville NY.) using diode lasers 402, 488 and 561. Serial optical sections were obtained with EZ-C1 computer software (Nikon Inc. Mellville, NY). Z series sections were collected at $0.3 \mu \mathrm{m}$ with a $40 x$ PlanApo lens and a scan zoom of 2 or with a $60 x$ Plan Apo objective and a scan zoom of 2, collected every $0.25 \mu \mathrm{m}$. Deconvolution measurements were performed with Elements (Nikon Inc. Mellville, NY) computer software. Five cells were outlined and analyzed per field.

\section{Xenograft studies to evaluate antitumor response of UR214-9}


NSG mice were implanted in their left flank with 1 million PANC-1 (HER2+, n=12), JIMT1 (number of animals=10) and SKOV-3 (number of animals=10) cells each in matrigel:media (1:1). Mice were randomized, identified with ear punches and subdivided into vehicle and treatment groups when tumors were found palpable. Both JIMT1 and SKOV-3 formed aggressive tumors within a week and were treated with vehicle or UR214-9 $(25 \mathrm{mg} / \mathrm{kg}$, IP, seven days a week). PANC-1 formed slow growing tumors and when tumors reached length exceeding $5 \mathrm{~mm}$, the treatment was started. A group of SKOV-3 cells were also treated with Herceptin or Herceptin+UR214-9. The vehicle formulation was: 40\% Hydroxypropyl-beta-cyclodextrin [Acros Organics] \& solutol HS15 (Sigma] in sterile water). $25 \mathrm{mg} / \mathrm{kg}$ equivalent of UR214-9 (1 uL=200ug in DMSO) was dissolved in $600 \mathrm{uL}$ PBS+400uL of the vehicle and vortexed to obtain a clear suspension. Tumor burden and animal weight was measured manually by digital calipers on weekly or biweekly routine. Tumor volume was calculated using the formula $1 / 2\left(L \times W^{\wedge} 2\right)$ where $L$ is a longest diameter and $W$ is the widest width. Statistical difference between the vehicle and treatment groups was analyzed by GraphPrism-8 software using one way annova. $\mathrm{P}<0.05$ was considered significant. Mice after the treatment period were euthanized and tumors were resected, weighed and frozen in liquid nitrogen. A portion of the tumors from the control and treatment groups were fixed in neutral buffered formaldehyde and paraffin embedded. 5uM thickness tissues slides were prepared for histochemistry.

\section{mRNA Sequencing}

The total RNA concentration was determined with the NanopDrop 1000 spectrophotometer (NanoDrop, Wilmington, DE) and RNA quality assessed with the Agilent Bioanalyzer (Agilent, Santa Clara, CA) ${ }^{35}$. The TruSeq Stranded mRNA Sample Preparation Kit (Illumina, San Diego, $\mathrm{CA})$ was used for next generation sequencing library construction per manufacturer's protocols. Briefly, mRNA was purified from 200ng total RNA with oligo-dT magnetic beads and fragmented. First-strand cDNA synthesis was performed with random hexamer priming followed by secondstrand cDNA synthesis using dUTP incorporation for strand marking. End repair and 3` adenylation was then performed on the double stranded cDNA. Illumina adaptors were ligated to both ends of the cDNA, purified by gel electrophoresis and amplified with PCR primers specific to the adaptor sequences to generate cDNA amplicons of approximately 200-500bp in size. The amplified libraries were hybridized to the Illumina single end flow cell and amplified using the cBot (Illumina, San Diego, CA). Single end reads of $75 \mathrm{nt}$ were generated for each sample using Illumina's NextSeq55036.

\section{Whole transcriptome data analysis}

Raw reads generated from the NovaSeq6000 sequencer were demultiplexed using bcl2fastq version 2.19.0. Quality filtering and adapter removal are performed using Trimmomatic-0.36 with the following parameters: "TRAILING:13 LEADING:13 ILLUMINACLIP:adapters.fasta:2:30:10 SLIDINGWINDOW:4:20 MINLEN:35" Processed/cleaned reads were then mapped to the Homo sapiens reference sequence (GRCh38, hg38) with STAR-2.6.0c given the following parameters: "--twopassMode Basic --runMode alignReads --genomeDir \$\{GENOME\} --readFilesIn \$\{SAMPLE\} --outSAMtype BAM SortedByCoordinate --outSAMstrandField intronMotif --outFilterlntronMotifs RemoveNoncanonical". The subread-1.6.1 package (featureCounts) was used to derive gene counts given the following parameters: "-s 2 -t exon -g gene_name". Differential expression analysis and data normalization was performed using DESeq2-1.16.1 with an adjusted p-value threshold of 0.05 within an R-3.4.1 environment. Heatmaps were created using the pheatmap R package ${ }^{37-39}$. 


\section{Data acquisition and statistical analysis}

The prognostic assessment of septin-2, -7 and -9 in the panel of different cancers was conducted using Human Protein Atlas tools. Alternatively, R2 genome.org tools were employed to determine the impact of septins enrichment on the survival prospects. $P$ values less than 0.05 were considered significant. The relative tumor sizes in the naïve vs treat groups were calculated using GraphPrism8 using one-way annova settings. P values less than 0.05 were considered significant.

\section{Discussion}

Data continue to emerge on the association of septins with malignancies, making identification of septin-targeted therapies crucial to block the aberrant septin functions in cancer cells. Considering FCF that essentially strengthens septin- $2^{40}$ as the starting point, we have developed UR214-9, a small molecule which dismantles septin-2 and -9 filamental assembly in cancer cells without killing the cells or altering the septins protein levels in the cells. To our best knowledge, this is the first description of a pharmacologic approach to disrupt septin's structure in cells. We considered that disrupting oligomeric septin filamental structures via UR214-9 treatment will be the key to impact cytokinesis and control the proliferation of cancer cells. Not only did the disruption of septin filaments by UR214-9 reduce the proliferation of pancreatic cancer cells (and of breast, ovarian endometrial, lung and kidney cancers; some data not shown) in vitro, xenografted tumors of breast, ovarian, pancreatic and lung malignancies (data not shown) treated with UR214-9 also showed reduction in tumor growth. Interestingly, the combination with Herceptin led to stronger control over HER2 positive SKOV-3 xenograft's growth (Figure-6D).

Enhancement in antitumor effects of Herceptin via co-treatment with UR214-9 in HER2 positive ovarian cancer xenograft model is stemming likely from the association of septin-2 with HER2. Septin-2 is shown to maintain HER2 signaling in cancer cells ${ }^{27}$. Septins protect and stabilize HER2 receptor at the plasma membrane of tumor cells to perpetuate the HER2 orchestrated oncogenic signaling and tumorigenesis ${ }^{27}$. It is anticipated that targeting septins can improve the survival rate of HER2 positive breast, pancreatic and other malignancies such as ovarian and lung. HER2 overexpression leads to aggressive breast malignancy and poor patient survival ${ }^{41}$. Current repertoire of therapies for HER2+ malignancies are inadequate. More than $60 \%$ of HER2+ breast cancer patients do not respond to trastuzumab treatment and resistance to the treatment develops rapidly in virtually all patients ${ }^{42}$. Further, the inability of trastuzumab to penetrate solid breast tumors to block secreted (truncated) forms of HER2, that promote resistance and metastasis, limits its usefulness in providing a complete and lasting control over HER2 orchestrated breast tumor growth ${ }^{43}$. Similarly, treating or preventing brain metastases in patients with HER2+ breast cancer is challenging ${ }^{44}$, particularly in the post-trastuzumab phase of treatment. About two-thirds of patients develop brain metastases despite control or response of their extracranial disease to trastuzumab ${ }^{45}$. Because trastuzumab does not penetrate the central nervous system, the brain may serve as a sanctuary site ${ }^{46}$. A blood-brain barrier (BBB) penetrant drug would be required to better control brain metastases in patients with HER2 + positive cancers. UR214-9 carries the structural attributes of small polar surface area signatures (calculated for UR214-9 $=53.49$ vs $<90$ required to cross BBB) that would facilitate penetration through the BBB. UR214-9, therefore, may improve outcomes of patients with brain metastases from HER2+ cancers.

Signaling associations of septins are not fully understood. To determine signaling association of septins and perturbations that UR214-9 treatment mounts, we conducted global rna-seq analyses of breast and pancreatic cancer cells treated with UR214-9 and, as a comparator, afatinib, a HER2 targeted therapy. As shown in Figure-7, afatinib treatment clearly had the most impact on the transcriptional profile of PANC-1 cells, while treatment with DMSO and UR214-9 did not have much effect on the transcriptome. The lack of differentially expressed 
genes between the UR214-9 treatment and control suggests that the mode of action of UR214-9 is non-transcriptional, and treatment with UR214-9 does not appear to elicit a gross transcriptional response.

Taken together, this study demonstrates that aberrant septins expression indicates poor prognoses among patients with cancer. UR214-9 is the first prototype of a small molecule that can induce septin-2 and -9 filamental catastrophe, a pharmacologic and cytoskeletal response of the cells not described before, to control cancer cell proliferation and tumor growth. Moreover, an important pharmacologic feature of UR214-9 is the benefits of limited off-target engagements. As shown in Figure-7, compared to afatinib, an EGFR targeted therapy that affected gene expression of over 1200 genes in JIMT-1 breast cancer cells, UR214-9 treatment even at 100-fold higher dose affected less than 20 genes significantly. Although UR214-9 is a close structural analog of FCF, UR214-9 differs significantly from FCF pharmacologically. While FCF is shown to strengthen septin-2, UR214-9 dismantles septin-2 and septin-9 filamental assembly. ICM scores calculated through molecular docking indicated greater binding affinity of UR214-9 with septin2:septin-2 dimer complex than FCF. To the best of our knowledge, other than FCF, which is clinically unfit due to the weak pharmacologic effects, off-target effects and functions associated with strengthening the septin-2 filaments, UR214-9 is the only septin modulator described so far that can dismantle septin's structural arrangement in nano molar concentrations (70nM-1uM). Given the preliminary antitumor response in breast, pancreatic, ovarian and lung cancer xenograft models (data not shown) and its therapeutic capabilities to significantly enhance the response of Herceptin in HER2 expressing xenograft tumors it is apparent that dismantling septins is an effective and clinically promising approach to prevent tumor growth, although doses, delivery formulations and frequencies of administrations have to be optimized, and a synergistic or at least an additive combinational agent has to be identified to achieve fuller control over the tumor growth. Based on the promising outcome in combination with Herceptin, our laboratory is currently evaluating the outcome of combination of UR214-9 with paclitaxel and Herceptin in breast cancer models to increase the clinical utilities of UR214-9.

Figures and figure legends: 

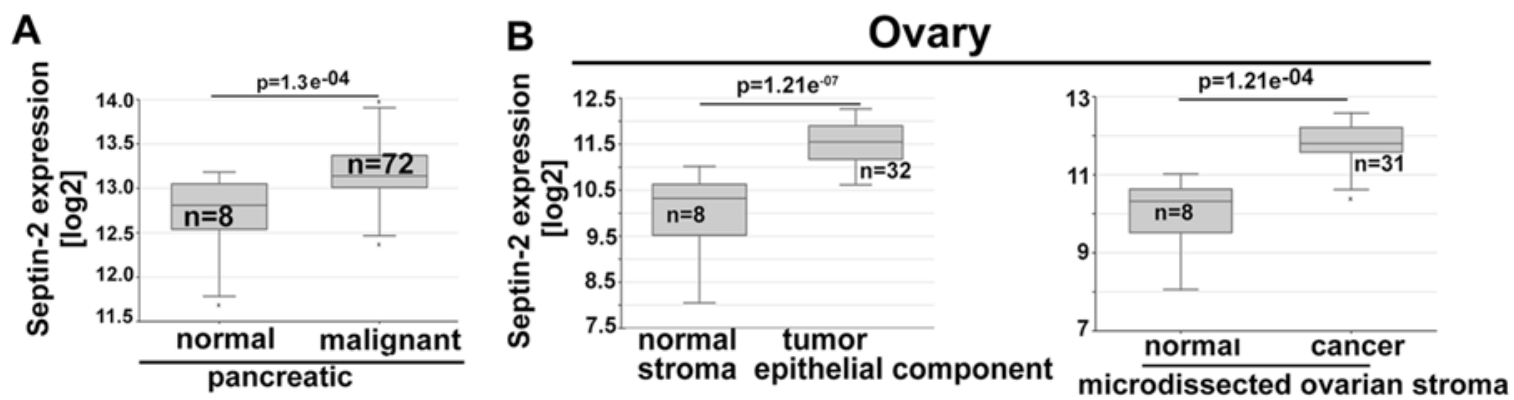

$c$
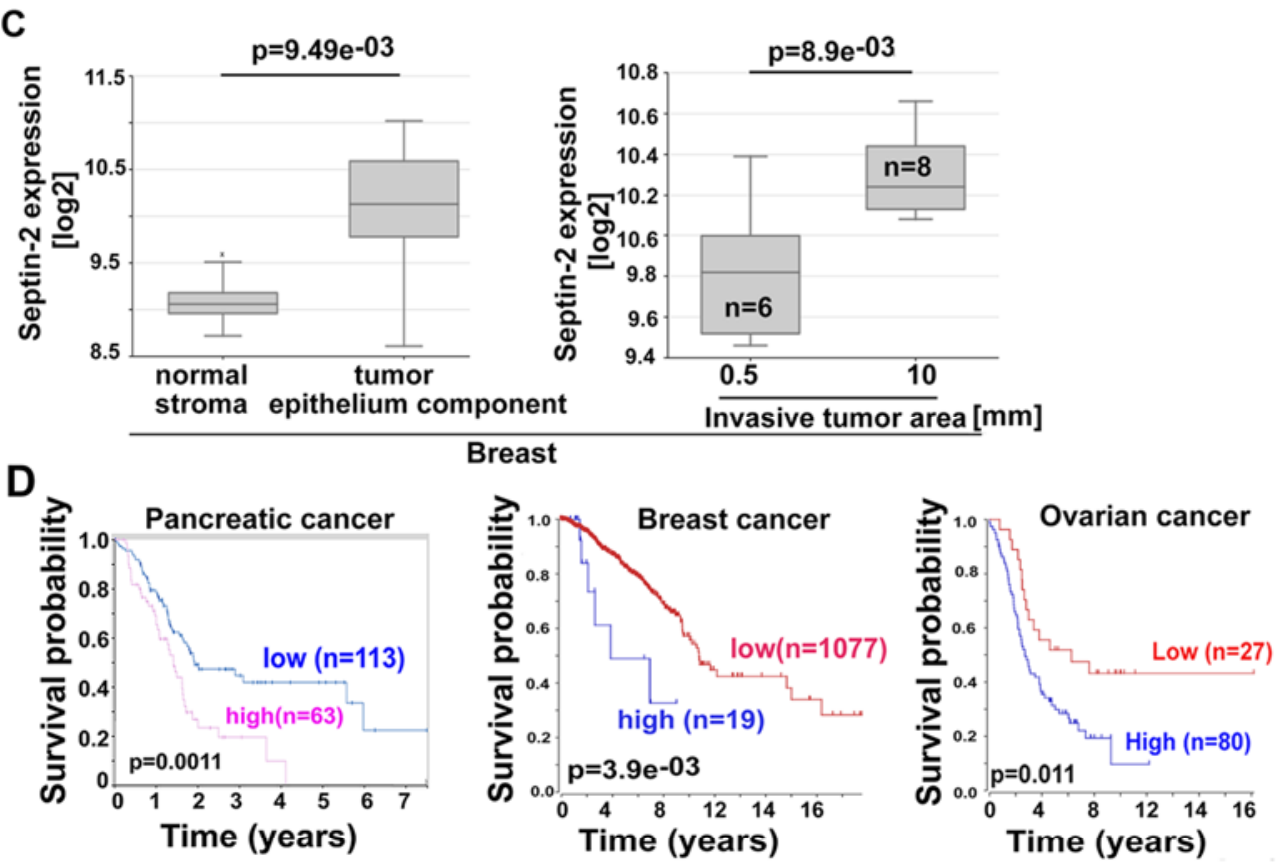

E

Septin-2
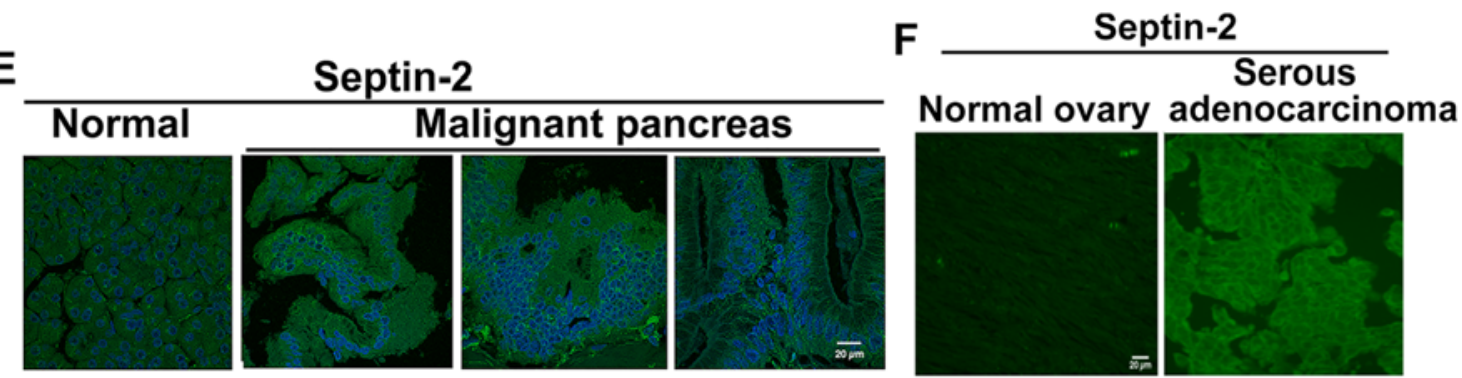

Figure-1: (A-C): Septin-2 expression in normal and malignant pancreatic, ovarian and breast cancer tissues were analyzed using the publicly accessible patient's tumor microarray data deposited on R2-Genomics Analysis and Visualization Platform (https://hgserver1.amc.nl/cgibin/r2/main.cgi). (D): Kaplan Meier survival analyses of the pancreatic, ovarian and breast cancer cancer patients using the data and tools available at the Human Protein Atlas or at R2- R2Genomics Analysis and Visualization Platform show that septin-2 enrichment correlates with decreased survival among pancreatic, breast and ovarian cancer patients. Septin-7 and -9 enrichment was associated with increased mortality as well (see supplementary Figure-1). (E): Pancreatic Tumor microarray from US Biomax Inc (catalog number: T142a) was stained with septin-2 antibody (Sigma Aldrich Inc. catalog number: HPA018481), followed by sourced- 
520 matched secondary antibody (DyLight-488, catalog number DI-1488, Vector laboratories Inc.). 521 Imaged were recorded as described in the methods section. Malignant tissues showed higher 522 septin-2 expression than tissues isolated from normal pancreas. (F): Malignant serous ovarian 523 cancer tissues showed increased septin-2 expression than normal ovaries. Ovarian tumor 524 microarray from US Biomax Inc (catalog OV241a) was stained with septin-2 antibody (Sigma 525 Aldrich catalog number: HPA018481) followed by a sourced-matched secondary antibody 526 (DyLight-488, catalog number DI-1488, Vector laboratories Inc.). Imaged were acquired as 527 described in method section. 
A

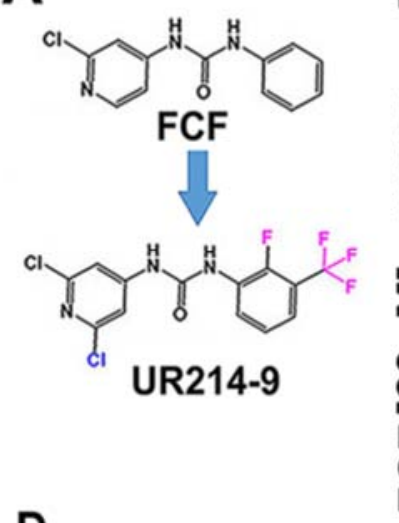

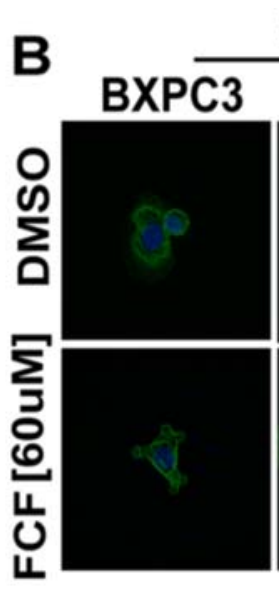

D

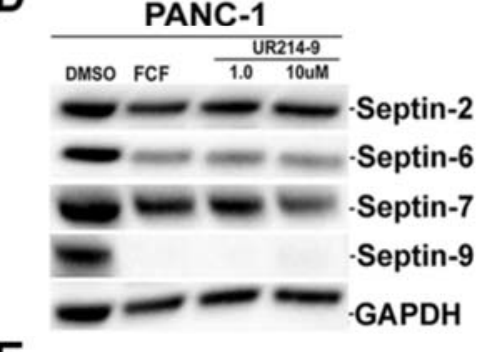

E

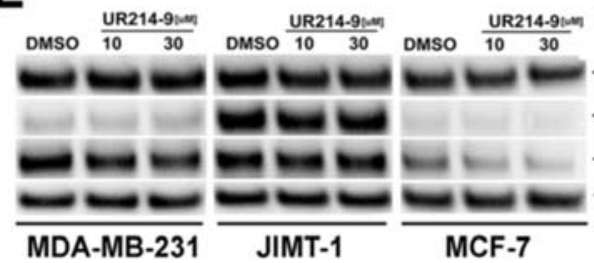

Septin-2
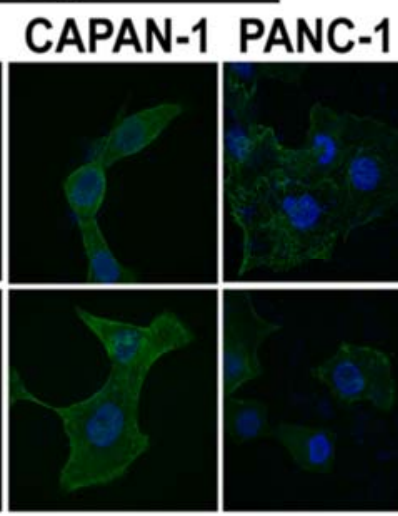

F

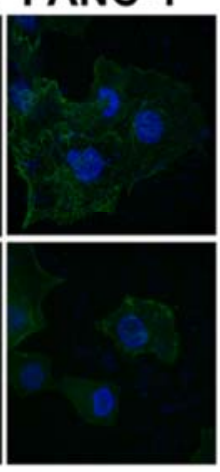

Septin-2

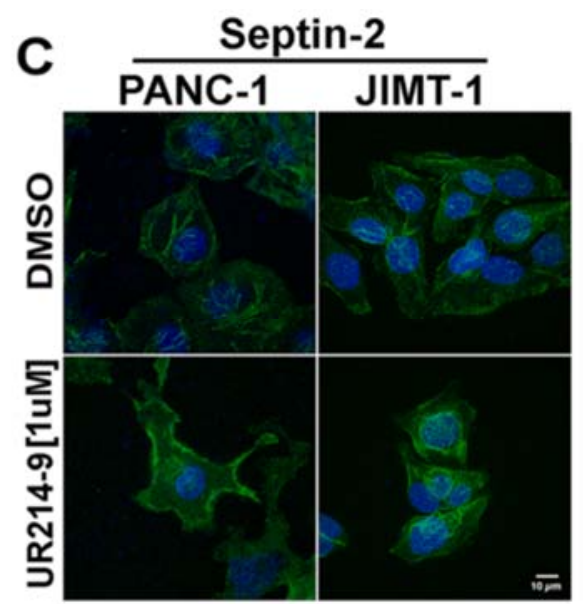

G

Figure-2: (A): Chemical structure of FCF and UR214-9. Structural changes to FCF leading to UR214-9 are shown in blue and pink color. (B): BXPC-3, CAPAN-1 and PANC-1 cells were seeded on glass slides and treated with DMSO or FCF(60uM) for 48 hours. The cells were fixed, processed and stained with validated septin-2 antibody (Sigma Aldrich Inc. Catalog number: HPA018481) and source matched secondary antibody (DyLight 488, Vector Laboratories Inc., catalog number: Dl-1488), and confocal images were recorded at 60x2 magnification. (C): PANC1 and JIMT-1 seeded on glass chamber slides cells were treated with DMSO (vehicle) or UR214$9(1 \mu \mathrm{M})$ for $48 \mathrm{hrs}$. Cells were fixed, permeabilized and stained with septin-2 antibody (Sigma Aldrich Inc. Catalog number: HPA018481) and DyLight 488 conjugated secondary antibody (Vector Laboratories Inc., catalog number: DI-1488), and confocal images were recorded at 60x2 magnification. (D): PANC-1 cells were seeded in $100 \mathrm{~mm} 3$ dishes and treated with DMSO, FCF $(60 \mu \mathrm{M})$, UR214-9 (1.0 and $10.0 \mu \mathrm{M})$ for 48 hours. Total cells lysates were immunoblotted and probed with the validated septin-2 (Sigma Aldrich Inc. Catalog number: HPA018481),6 (Sigma Aldrich Inc. Catalog number: HPA005665), 7(Sigma Aldrich Inc. Catalog number: HPA029524) and -9 (Sigma Aldrich Inc. Catalog number: HPA042564) antibodies. (E): MDA-MT-231, JIMT-1 and MCF-7 cells seeded in 100mm3 petri dishes were treated with DMSO or UR21409(10 and $30 \mu \mathrm{M})$ for 48 hours. The total cell lysates were immunoblotted and probed with validated septin2 (Sigma Aldrich Inc. Catalog number: HPA018481), 6 (Sigma Aldrich Inc. Catalog number: HPA005665) and -9 (Sigma Aldrich Inc. Catalog number: HPA042564) antibodies. (F-G): SKOV3 ovarian cancer cells seeded on glass chamber slides cells were treated with DMSO (vehicle) or UR214-9 $(1 \mu \mathrm{M})$ for $48 \mathrm{hrs}$. Cells were fixed, permeabilized and stained with validated septin-2 (Sigma Aldrich Inc. Catalog number: HPA018481),6 (Sigma Aldrich Inc. Catalog number: 
569 HPA005665), 7(Sigma Aldrich Inc. Catalog number: HPA029524) and -9 (Sigma Aldrich Inc. 570 Catalog number: HPA042564) antibodies followed by DyLight 488 conjugated secondary 571 antibody (Vector Laboratories Inc., catalog number: DI-1488), and confocal images were recorded 572 at $60 \times 2$ magnification. 


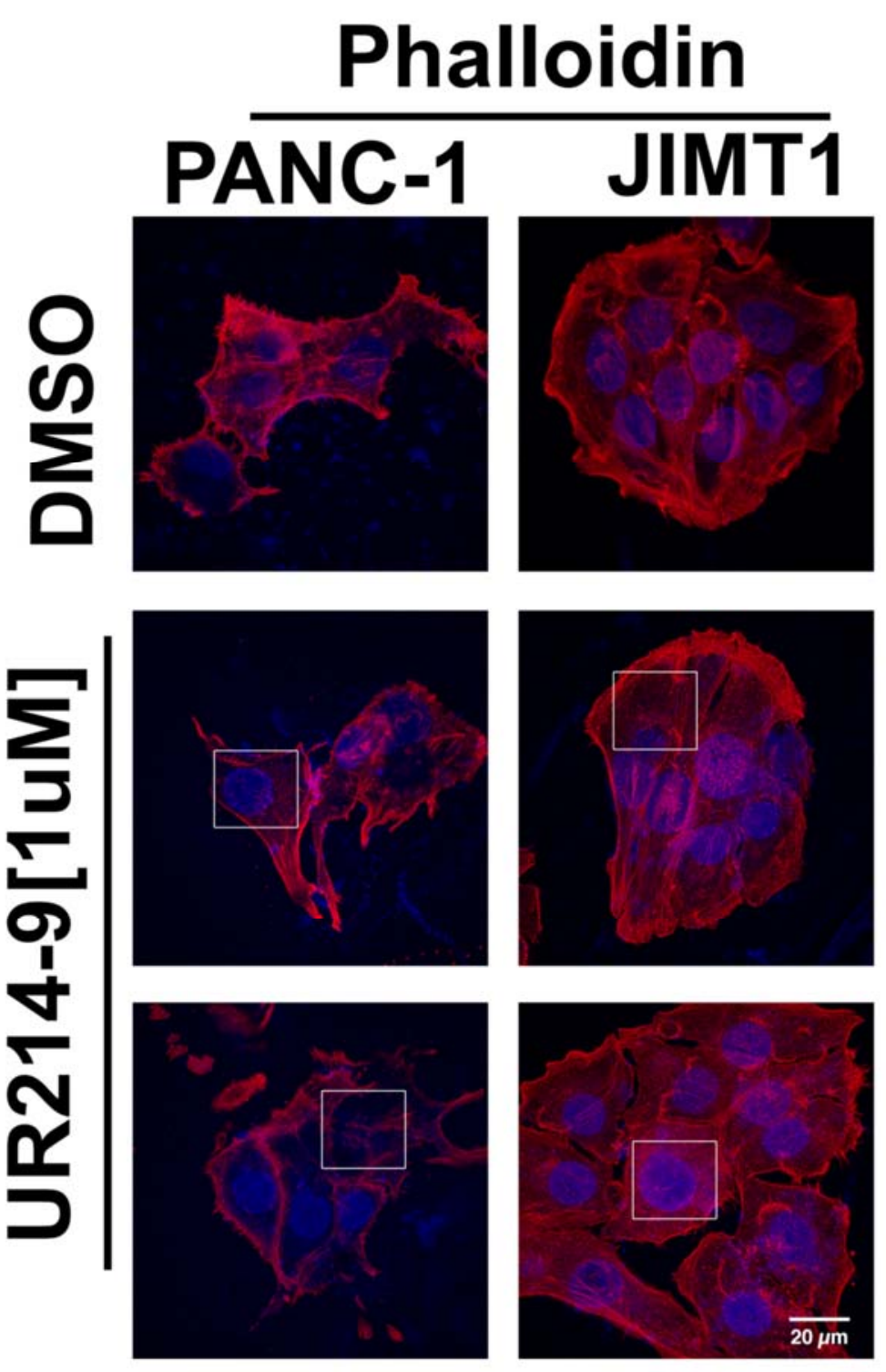

Figure-3: PANC-1 pancreatic cancer cells and JIMT-1 breast cancer cells were treated with vehicle or UR214-9 $(1 \mu \mathrm{M})$ for 48-hour duration, fixed, permeabilized and stained with PhalloidinTRITC (ECM Biosciences Inc., catalog number: PF7551). Confocal images were recorded at 60x2 magnification. Areas of interest are shown by white boxes. 
A

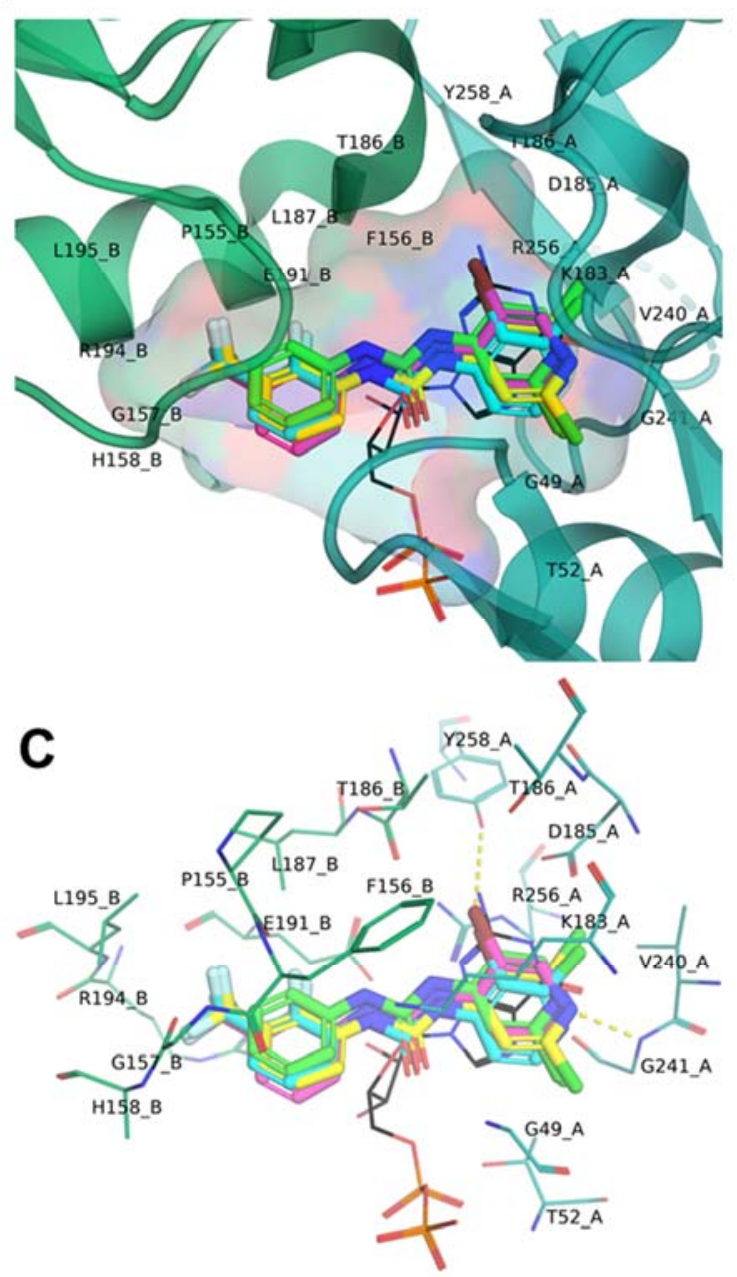

B

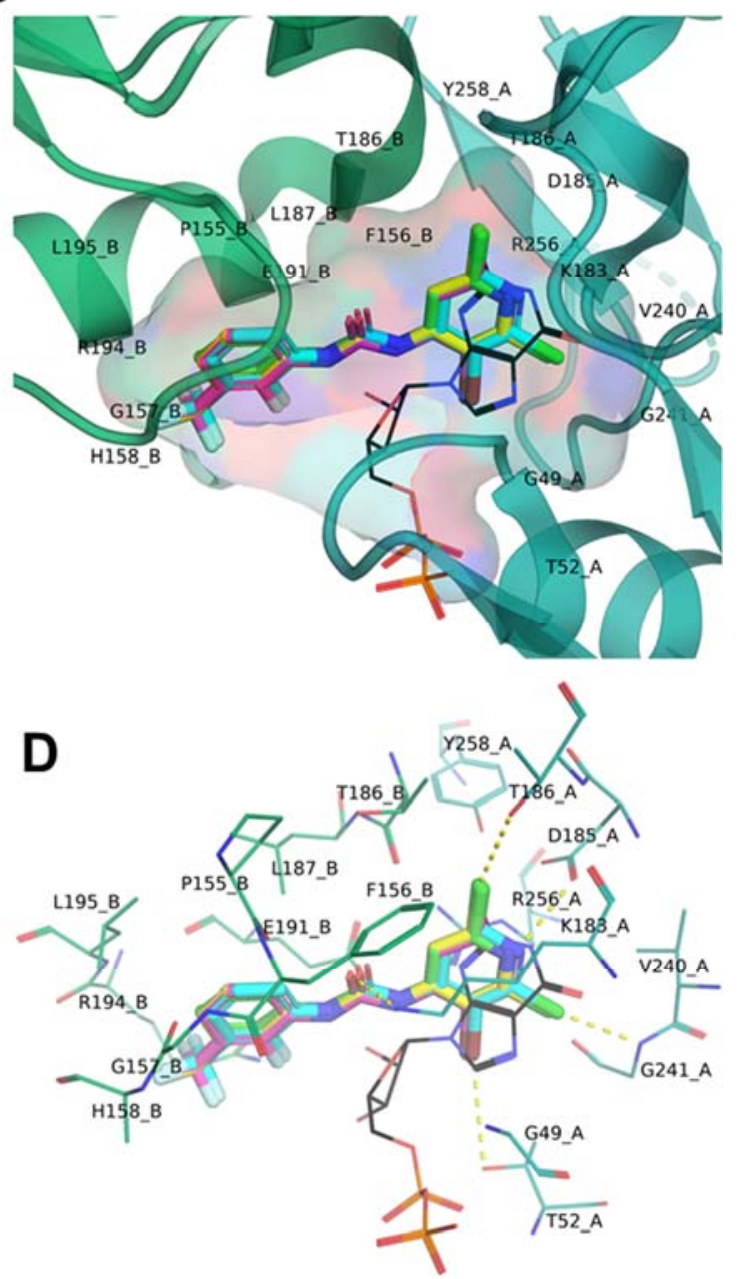

Fogure-4: (A-B): Septin-2:septin-2 dimer complex was docked with FCF, UR214-8, UR214-9 and UR214-10 using Molsoft's ICM software package (v. 3.8-7). Compounds were docked into the nucleotide binding site of PDB ID 2QNR, which is the highest quality structure of a septin-2 dimer complex available [33]. Receptor preparation (based on the GDP binding site in chain A) and ligand construction was performed within ICM using standard settings. The compounds were docked with the "dock table" functionality, with a setting for effort of 2.0 and 20 poses per compound. (C-D): Amino acid residues interacting with UR214-9 in GDP binding domain are shown. 


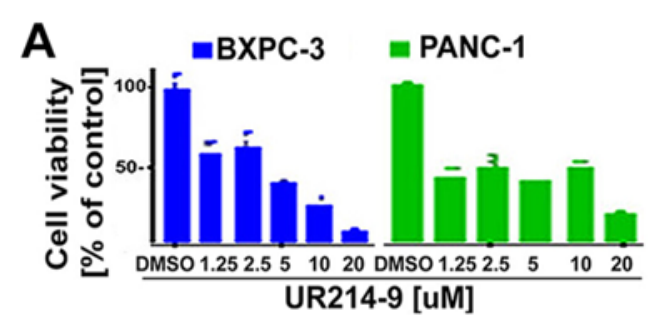

C

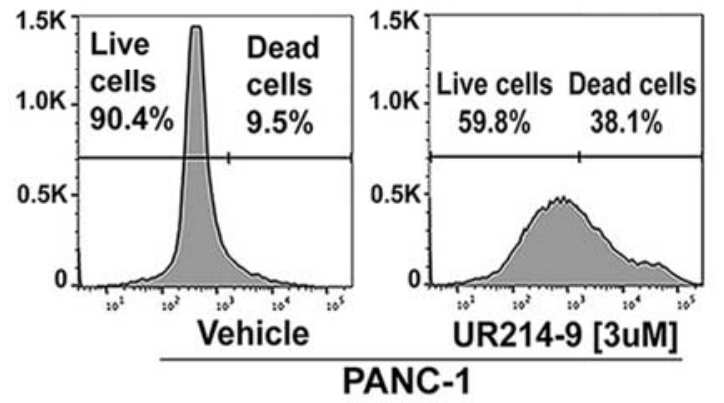

E

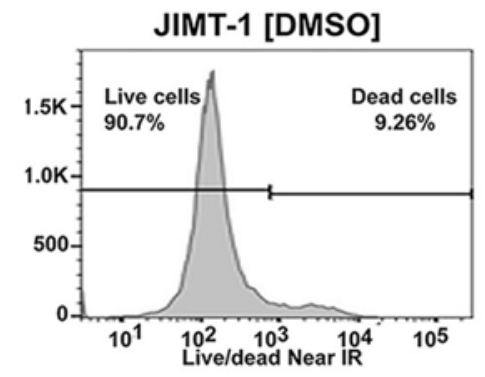

F

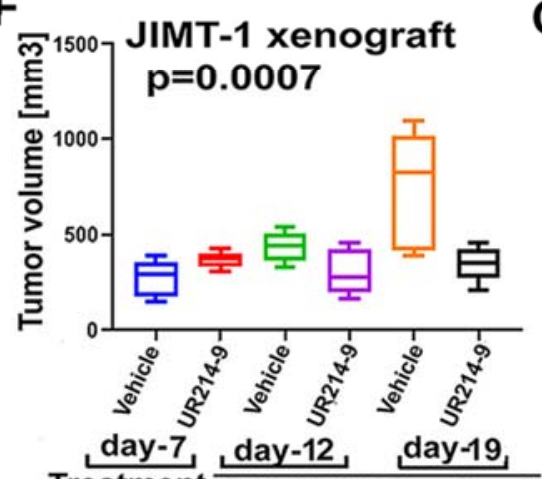

Treatment
begins

G

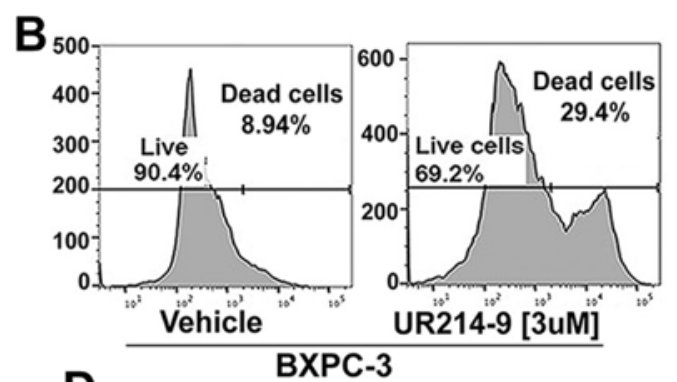

D
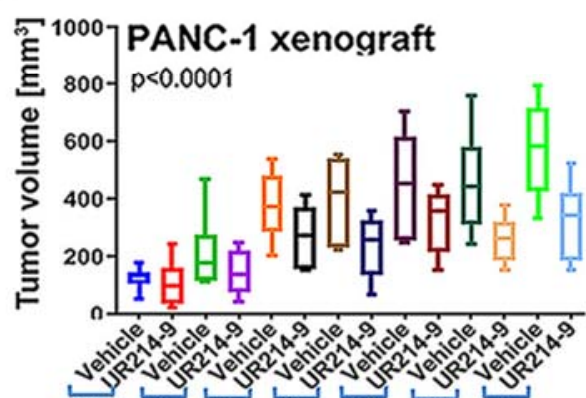

$\uparrow$ Days since tumor incoulation

Treatment UR214-9[25mg/kg, IP]
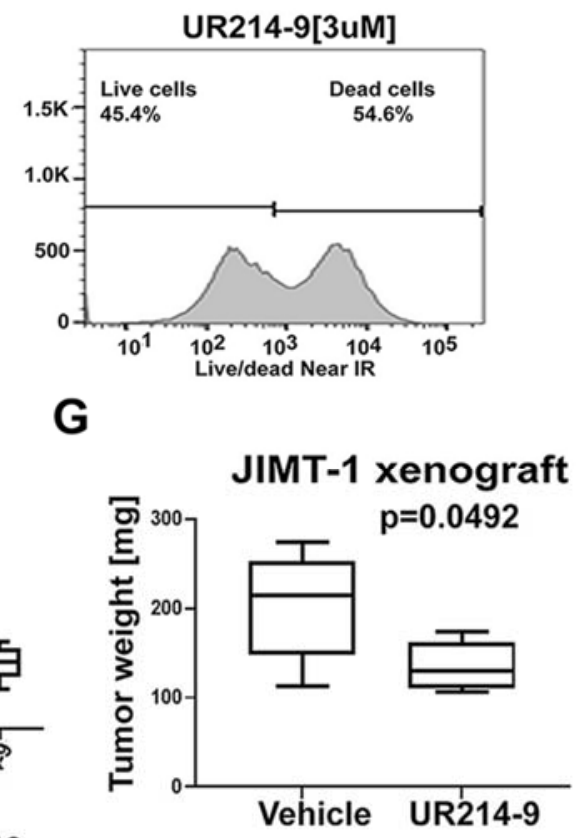

Figure-5: (A): Cell viability of PANC-1 and BXPC-3 cells treated with UR214-9 (DMSO, 1.25, 2.5, 5,10 and $20 \mathrm{uM}$ ) for 72 hours. The cell viability of the treated groups in comparison with DMSO group was assessed by use of MTS assay (Promega Corp. catalog number: G3580) and absorbance was read at 490nM using BioRad microplate reader. (B-C): PANC-1 and BXPC-3 cells were treated with UR214-9(3uM) or DMSO for 48 hours. The cells were stained with Live/dead near IR dye and the live and dead population in vehicle and control group was estimated by flow-cytometry. (D): NSG mice $(n=10)$ were inoculated with PANC-1 cells (1 
million/animal). Once tumors were palpable, mice were divided into two groups of $n=5$ each and treated with vehicle or UR214-9 (25mg, M-F, IP, once daily) for 52 days. The tumor sizes were measured periodically. (E): JIMT1 cells were treated with UR214-9(3uM) or DMSO for 48 hours. The cells were stained with Live/dead near IR dye and the live and dead population in vehicle and control group was estimated by flow-cytometry. $(\mathbf{F})$ : NSG mice $(n=10)$ were inoculated with JIMT1 cells ( 1 million/animal). Once tumors were palpable, mice were divided into two groups of $n=5$ each and treated with vehicle or UR214-9 (25mg, M-F, IP, once daily) for 28 days. The tumor sizes were measured periodically. (G): Tumors from both animals of the control and treatment groups were harvested and weighed on a calibrated balance. Statistical analysis was carried out using GraphPad Prism 8 software. A P value less than 0.05 was considered significant. 

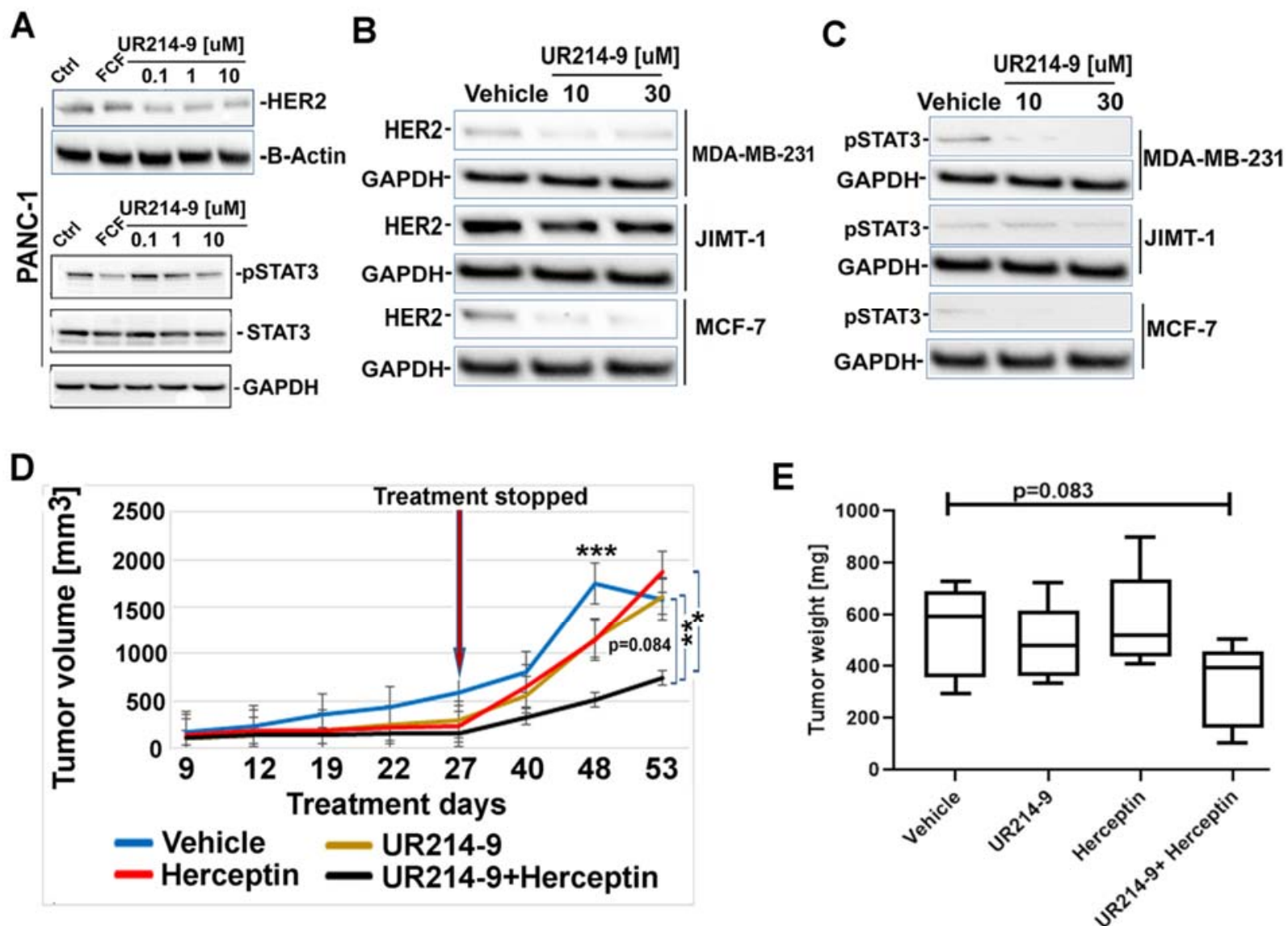

710

711

712

713

714

715

716

717

718

719

720

721

722

723

724

725

726

727

728

729

730

731

732

Figure-6: (A-B): Adhered BXPC-3, and PANC-1 cells were treated with FCF(60uM) and UR2149 (DMSO, 0.1, 1.0 and 10uM) for 48 hours. Cells were lysed and immunoblotted with HER2 (Cell Signaling Technology Inc., catalog number: 4290); pSTAT-3 (Cell Signaling Technology Inc., catalog number: 9145p), STAT-3 (Cell Signaling Technology Inc., catalog number: 4904), AKT (Cell Signaling Technology Inc., catalog number: 9272) and GAPDH antibodies (Cell Signaling Technology Inc., catalog number: 2118s). (C): MDA-MB-231, JIMT-1 and MCF-7 cells were treated with DMSO or UR214-9 $(10$ and $30 \mu \mathrm{M})$ for 24 hours. The cells were lysed, immunoblotted and probed with HER2 (Cell Signaling Technology Inc, catalog number: 4290) and phosphoSTAT-3 (Cell Signaling Technology Inc., catalog number: 9145p) antibodies. (D): HER2 expressing SKOV-3 cells (500,000 cells/animals) were implanted in the right flanks of NSG mice subcutaneously. When palpable, mice in groups ( $n=5$ each) were treated with vehicle, UR214-9 $(25 \mathrm{mg} / \mathrm{kg}, \mathrm{M}-\mathrm{F}$, I.P.), Herceptin (10 mg/kg, M, I.P.). Fourth group was treated with both UR214-9 $(25 \mathrm{mg} / \mathrm{kg}, \mathrm{M}-\mathrm{F}$, I.P.) and Herceptin $(10 \mathrm{mg} / \mathrm{kg}, \mathrm{M}$, I.P). Tumor sizes were measured on regular intervals using a digital caliper. Longest length and width were recorded. Tumor volumes was calculated using formula $\left(L^{*} W^{\wedge} 2\right)^{*} 0.5$ where $L$ represents longest diameter and $W$ stands for width of the tumors measured through a digital caliper. Treatment was stopped on day- $27^{\text {th }}$ and tumor sizes were measured on the days indicated. On the day- $53^{\text {rd }}$ since inoculation, mice were euthanized and tumors were harvested. Tumor weights were recorded using a calibrated balance. The statistical analyses were performed using Graph Prims 8.1.1.T-test analyses among groups were performed using Graph Prism 8.1.1. version and $p<0.05$ was considered significant. Day22: vehicle vs UR214-9: $p=0.0035^{* *}$; vehicle vs Herceptin: $p=0.0011^{* *}$; vehicle vs UR2149+Herceptin: $p=0.0001^{* * *}$; UR214-9 vs UR214-9+Herceptin: $p=0.0059^{* *}$; Herceptin vs UR214- 
bioRxiv preprint doi: https://doi.org/10.1101/2020.02.19.954529; this version posted February 20, 2020. The copyright holder for this preprint (which was not certified by peer review) is the author/funder. All rights reserved. No reuse allowed without permission.

733 9+Herceptin: $p=0.049^{*}$. Day-27: vehicle vs UR214-9: $p=0.0004^{* * *}$; vehicle vs Herceptin: $734 p=0.0002^{* * *}$; vehicle vs UR214-9+Herceptin: $p<0.0001^{* * * *}$.

735

736

737

738

739

740

741

742

743

744

745

746

747

748

749

750

751

752

753 


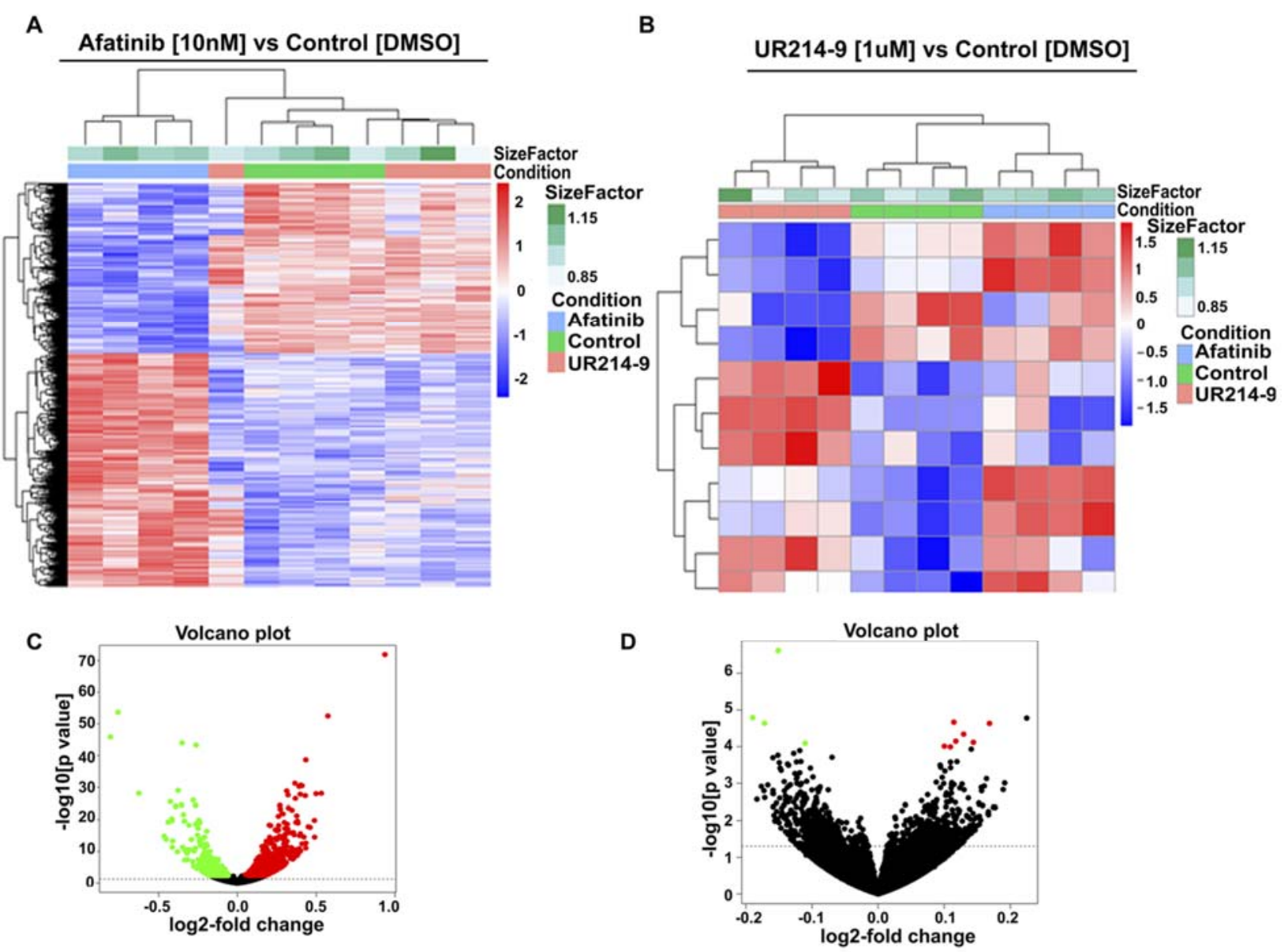

Figure-7: Hierarchically clustered heat map of mRNA expression for 1234 significantly differentially expressed genes (BH adjusted p-value < 0.05) in the JIMT-1 breast cancer cells treated with afatinib compared to control (A) and associated volcano plot (C). Hierarchically clustered heat map of mRNA expression for 11 significantly differentially expressed genes $(\mathrm{BH}$ adjusted p-value < 0.05) in the JIMT-1 breast cancer cells treated with UR214-9 compared to control (B) and associated volcano plot (D). Heatmap color key represents row scaling of the rLog transformed expression values. The volcano plots have horizontal lines at $p$-value 0.05 and individual genes/dots are colored red when the adjusted $p$-value is $>=0.05$ and the fold-change is $>0$ and green when the fold-change is $<0$. 


\section{Supplementary Figures:}
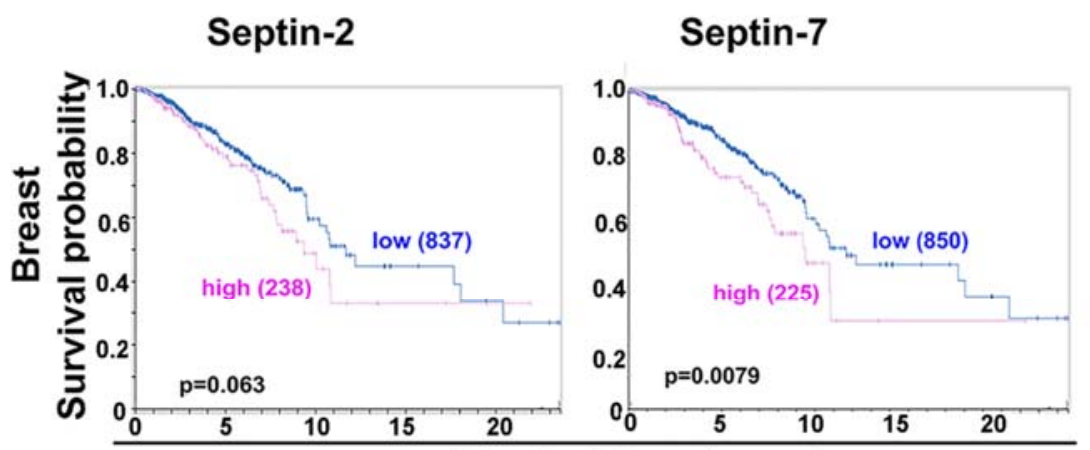

Survival time (years)
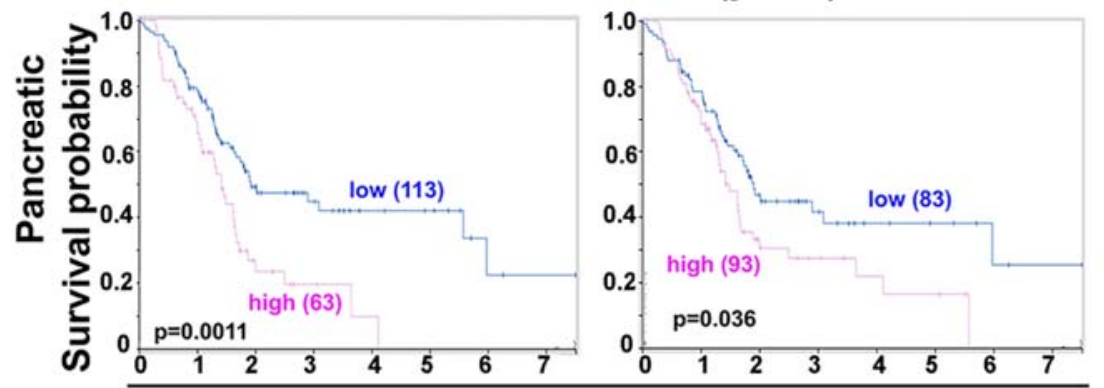

Survival time (years)
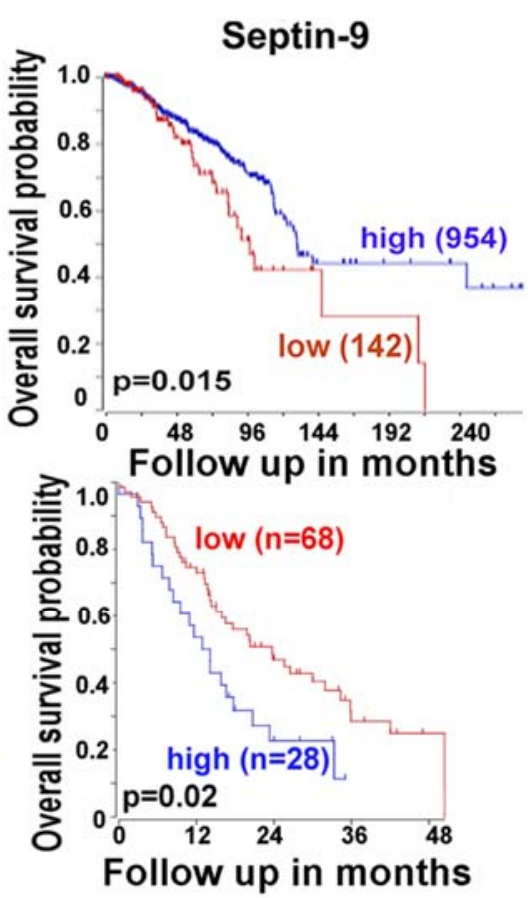

Supplementary Figure-1: Survival probabilities of patients diagnosed with breast and pancreatic, was correlated with septin-2 (left), septin-7 (middle) and septin-9 (right) gene expression using the data and tools available at the Human Protein Atlas ( https://www.proteinatlas.org/) or R2Genomics Analysis and Visualization Platform (https://hgserver1.amc.nl/cgi-bin/r2/main.cgi). P values less than 0.05 were considered significant. 
A
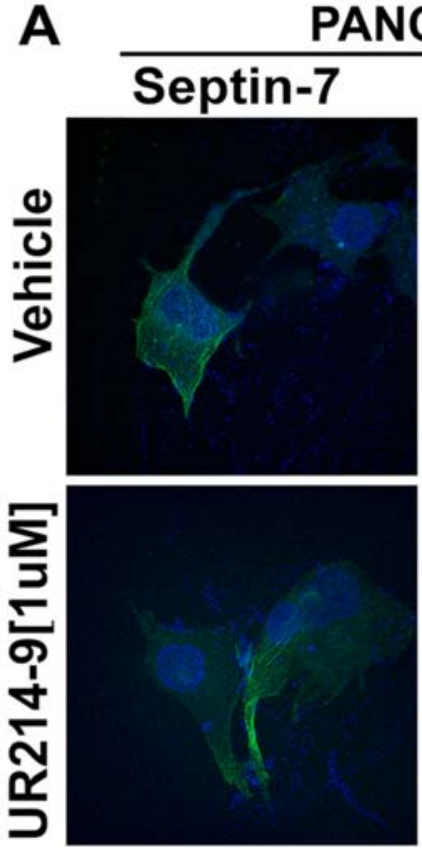

B Septin-9
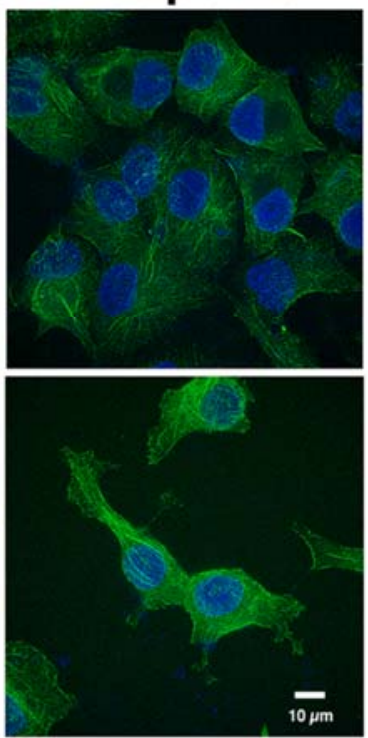
$\begin{array}{ll}\text { JIMT-1 cells } \\ \text { Septin-7 } & \text { Septin-9 }\end{array}$
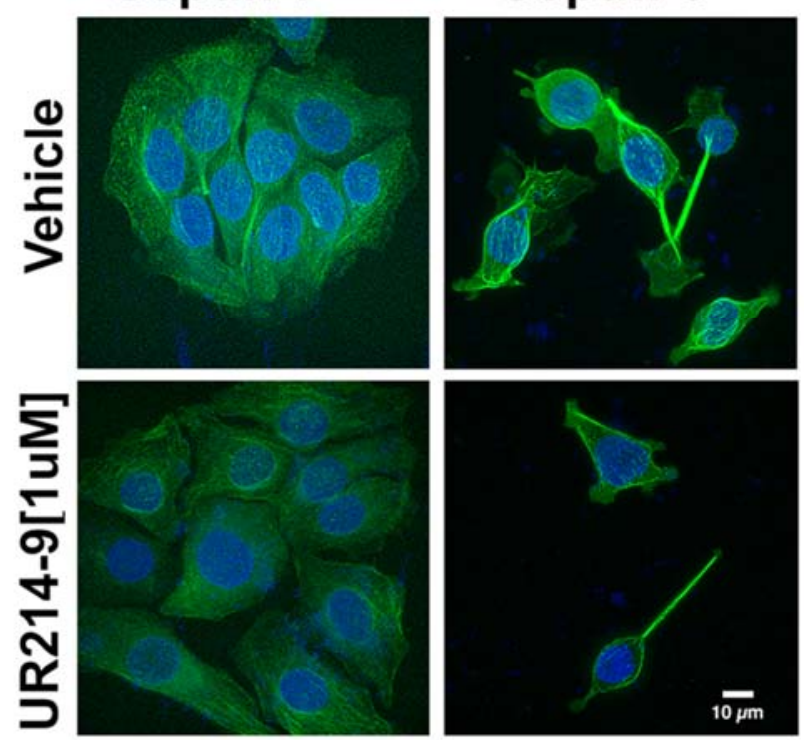

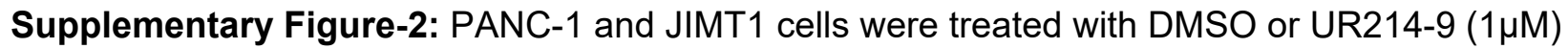
for $48 \mathrm{hrs}$. Cells were fixed, permealized and stained with septin-7 and -9 antibodies (Sigma Aldrich Inc., catalog number: HPA029524, HPA042564) and DyLight 488 conjugated secondary antibody (Vector Laboratories Inc., catalog number: DI-1488), and confocal images were recorded

800 at $60 \times 2$ magnification.

801

802

803 
804

805

806

807

808

809

810

811

812

813

814

815

816

817

818

819

820

821

822

823

824

825

826

827

828

829

830

831
A
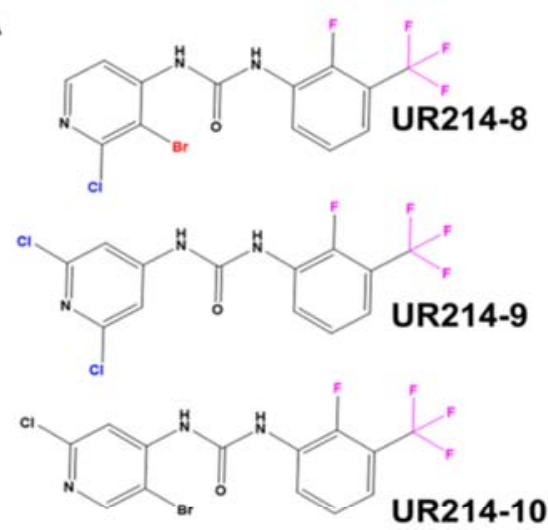

B
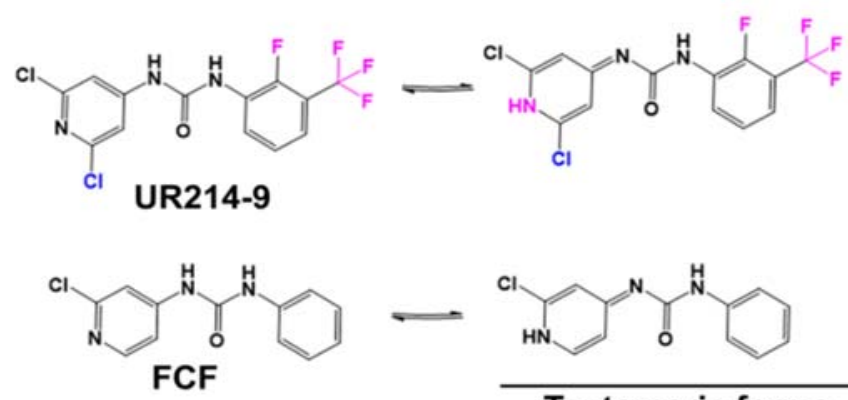

Tautomeric forms

Supplementary Figure-3: (A): Chemical structures of UR214-9 analogs employed in molecular docking. (B): Possible tautomeric forms of UR214-9 and FCF. 
A

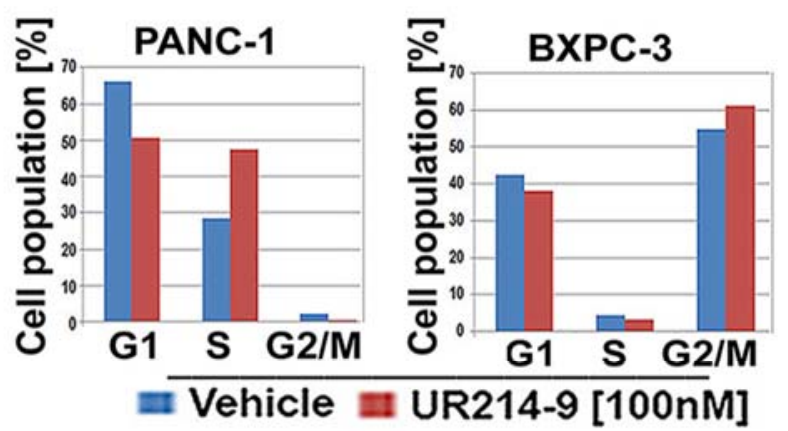

B

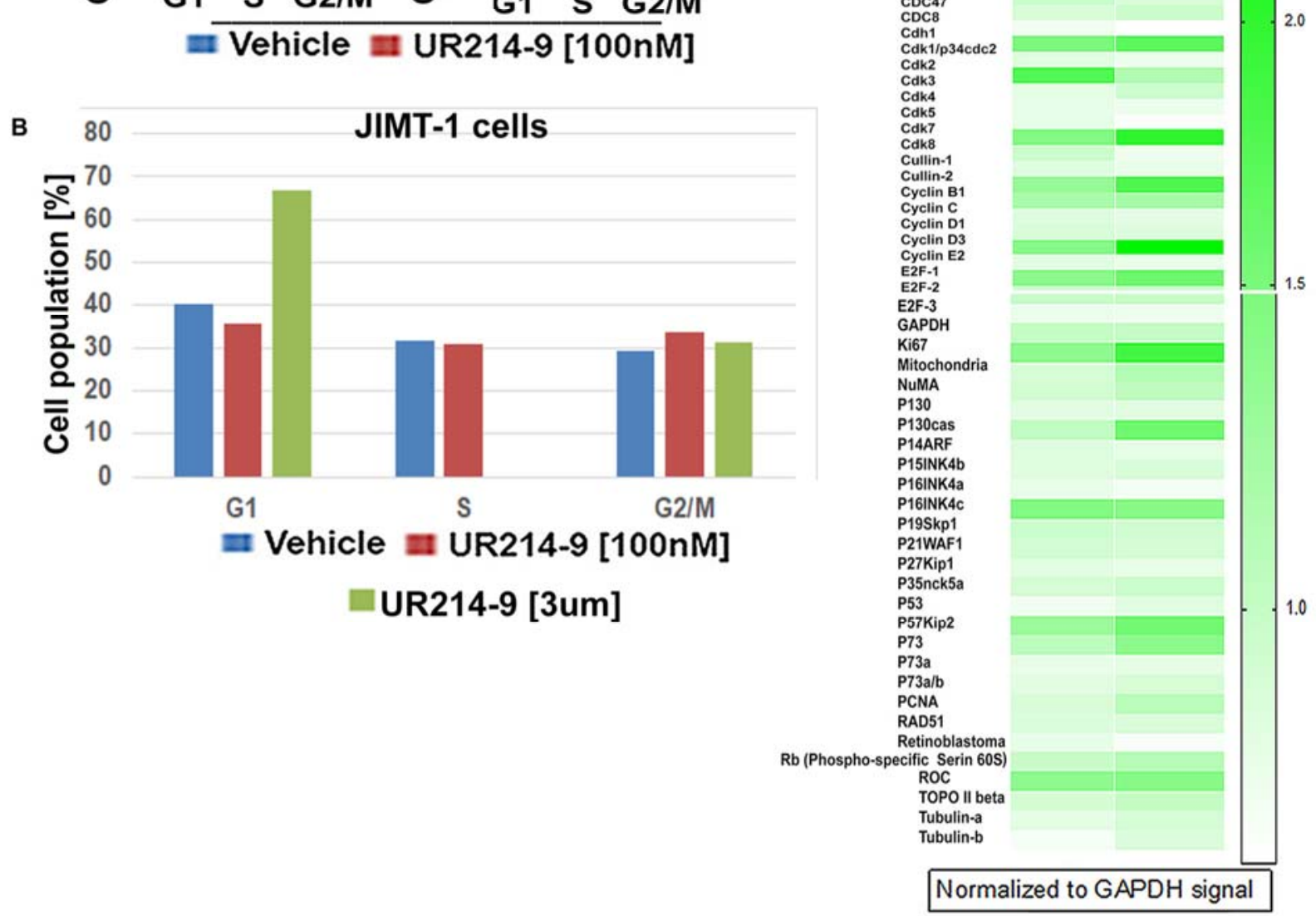

Supplementary Figure-4: (A): PANC-1 and BXPC-3 cells were treated with sub-cytotoxic concentrations of UR214-9 (100nM for 48 hours). Cells were fixed, permeabilized and stained with pre-formulated PI-RNase solution (Cell Signaling Technology, catalog number 4087s). DNA content was measured using flow-cytometry and cell cycle distribution was analyzed by flowjo or

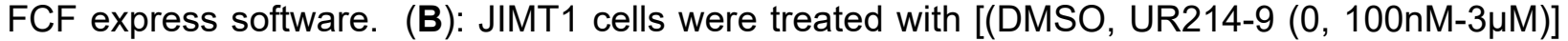
for 48 hours, fixed, permeabilized and stained with pre-formulated PI-RNase solution (Cell Signaling Technology, catalog number 4087s). DNA content was measured using flow-cytometry and cell cycle distribution was analyzed by flowjo software. (C): PANC-1 cells were treated with DMSO or UR214-9 (100nM) or $3 \mu \mathrm{M}$ for 48 hours. The cells were lysed and total protein was isolated using the buffer available in FullMoon Bioscience Cell Cycle Antibody array kit (catalog number: ASC058). The proteins isolated from the vehicle and treatment groups were applied on the antibody array, and processed and developed per the manufacturer's instructions. The photons were read using the array Image Quantification and Analysis services of FullMoon BioSciences (Catalog number SDA01-ACC058) and normalized to GAPDH. Cullin-3, GSK3b and p19ARF followed by Pan 14.3.3 were the most upregulated proteins in the treatment groups. Heatmap shows fold-changes in protein expressions. 


\section{Availability of data and UR214-9:}

The complete set of in vitro and in vivo results, rna-seq and western blot data are available from the corresponding author upon request. Similarly, reasonable quantities of UR214-9 will be freely made available for research and studies upon request.

\section{Reference}

1. Dolat, L., Hunyara, J.L., Bowen, J.R., Karasmanis, EP., Elgawly, M., Galkin, V.E., Spiliotis, E.T. Septins promote stress fiber-mediated maturation of focal adhesions and renal epithelial motility. J Cell Biol. 207,225-35(2014).

2. Mostowy, S. and P. Cossart, Septins: the fourth component of the cytoskeleton. Nat Rev Mol Cell Biol. 13,183-94(2012).

3. Tokhtaeva, E., Capri, J., Marcus, E.A., Whitelegge, J.P., Khuzakhmetova, V., Bukharaeva, E., Deiss-Yehiely, N., Dada, L.A., Sachs, G., Fernandez-Salas, E, Vagin, O. Septin dynamics Septin dynamics are essential for exocytosis. J Biol Chem. 290,5280-97(2015).

4. Kartmann, B. and D. Roth, Novel roles for mammalian septins: from vesicle trafficking to oncogenesis. J Cell Sci. 114(Pt 5),839-44 (2001).

5. Caudron, F. and Y. Barral, Septins and the lateral compartmentalization of eukaryotic membranes. Dev Cell. 16,493-506(2009).

6. Bridges, A.A. and A.S. Gladfelter, Septin Form and Function at the Cell Cortex. J Biol Chem. 290,17173-80(2015).

7. Pagliuso, A., Tham, T.N., Stevens, J.K., Lagache, T., Persson, R., Salles, A., OlivoMarin, JC, Oddos, S., Spang, A., Cossart, P., Stavru, F. A role for septin 2 in Drp1mediated mitochondrial fission. EMBO Rep. 17,858-73(2016).

8. Sirianni, A., Krokowski, S., Lobato-Márquez, D., Buranyi, S., Pfanzelter, J., Galea, D., Willis, A., Culley, S., Henriques, R., Larrouy-Maumus, G., Hollinshead, M., SanchoShimizu V., Way, M., Mostowy, S. Mitochondria mediate septin cage assembly to promote autophagy of Shigella. EMBO Rep. 17,1029-43(2016).

9. Mostowy, S., Bonazzi, M., Hamon, M.A., Tham, T.N., Mallet, A., Lelek, M., Gouin, E., Demangel, C., Brosch, R., Zimmer, C., Sartori, A., Kinoshita, M., Lecuit, M., Cossart, P. Entrapment of intracytosolic bacteria by septin cage-like structures. Cell Host Microbe. 8,433-44(2010).

10. Traikov, S., Stange, C., Wassmer, T., Paul-Gilloteaux, P., Salamero, J., Raposo, G., Hoflack, Bernard. Septin6 and Septin7 GTP binding proteins regulate AP-3- and ESCRT-dependent multivesicular body biogenesis. PLoS One. 9(11), e109372(2014).

11. Dolat, L. and E.T. Spiliotis, Septins promote macropinosome maturation and traffic to the lysosome by facilitating membrane fusion. J Cell Biol. 214,517-27(2016).

12. Russell, S.E., Hall, P.A. Do septins have a role in cancer? Br J Cancer. 93,499503(2005).

13. Hall, P.A., Russell, S.E. The pathobiology of the septin gene family. J Pathol. 204,489505(2004).

14. Cerveira, N., S. Bizarro, and M.R. Teixeira, MLL-SEPTIN gene fusions in hematological malignancies. Biol Chem. 392,713-24(2011).

15. Connolly, D., et al., Septin roles in tumorigenesis. Biol Chem. 392,725-38 (2011).

16. Angelis, D. and E.T. Spiliotis, Septin Mutations in Human Cancers. Front Cell Dev Biol. 4:122 (2016).

17. Dolat, L., Q. Hu, and E.T. Spiliotis, Septin functions in organ system physiology and pathology. Biol Chem. 395,123-41(2014). 
18. Marttinen, M., Kurkinen, K.M., Soininen, H., Haapasalo, A., Hiltunen, M. Synaptic dysfunction and septin protein family members in neurodegenerative diseases. Mol Neurodegener. 10,16 (2015).

19. Calvo, F., Ranftl, R., Hooper, S., Farrugia, A.J., Moeendarbary, E., Bruckbauer, A., Batista, F., Charras, G., Sahai, E. Cdc42EP3/BORG2 and Septin Network Enables Mechano-transduction and the Emergence of Cancer-Associated Fibroblasts. Cell Rep. 13,2699-714(2015).

20. https://hgserver1.amc.nl/cgi-bin/r2/main.cgi

21. Uhlén, M et al. Tissue-based map of the human proteome. Science 347,1260419 (2015).

22. Schmidt, K., Nichols, BJ. Functional interdependence between septin and actin cytoskeleton. BMC Cell Biol.12,43 (2004).

23. Angelis, D., et al., In silico docking of forchlorfenuron (FCF) to septins suggests that FCF interferes with GTP binding. PLoS One. 9,e96390 (2014).

24. Pintard, L., Willems, A., Peter, M. Cullin-based ubiquitin ligases: Cul3-BTB complexes join the family. EMBO J. 23,1681-7(2004).

25. Kumar, V., Abbas, A., Aster, J. Robbins basic pathology. Philadelphia: Elsevier/Saunders. 2013; p. 697. ISBN 9781437717815.

26. Buza, N., Roque, DM., Santin, AD. "HER2/neu in Endometrial Cancer: A Promising Therapeutic Target With Diagnostic Challenges". Archives of Pathology \& Laboratory Medicine.138,343-50(2014).

27. Rüschoff, J., Hanna, W., Bilous, M., Hofmann, M., Osamura, R.Y., Penault-Llorca, F., van de Vijver, M,, Viale, G. "HER2 testing in gastric cancer: a practical approach". Modern Pathology. 25:637-50(2012).

28. Ruiz-Saenz, A., Dreyer, C., Campbell, M.R., Steri, V., Gulizia, N., Moasser, M.M. HER2 Amplification in Tumors Activates PI3K/Akt Signaling Independent of HER3. Cancer Res.78,3645-3658(2018).

29. Marcus, E.A., Tokhtaeva, E., Turdikulova, S., Capri, J., Whitelegge, J.P., Scott, D.R., Sachs, G., Berditchevski, F., Vagin, O. Septin oligomerization regulates persistent expression of ErbB2/HER2 in gastric cancer cells. Biochem J. 473,1703-18 (2016).

30. James, N.E., Cantillo, E., Yano, N., Chichester, C.O., DiSilvestro, .PA., Hovanesian, V., Rao, R.S.P., Kim, K.K., Moore, R.G., Ahsan, N., Ribeiro, J.R. Septin-2 is overexpressed in epithelial ovarian cancer and mediates proliferation via regulation of cellular metabolic proteins. Oncotarget. 10,2959-2972(2019).

31. DeFazio-Eli, L., Strommen, K., Dao-Pick, T., Parry, G., Goodman, L., Winslow, J. Quantitative assays for the measurement of HER1-HER2 heterodimerization and phosphorylation in cell lines and breast tumors: applications for diagnostics and targeted drug mechanism of action. Breast Cancer Res.13(2),R44 (2011).

32. English, D.P., Roque, D.M., Santin, A.D. HER2 expression beyond breast cancer: therapeutic implications for gynecologic malignancies. Mol. Diagn. Ther. 17,85-99 (2013).

33. Chung, S.S., Giehl, N., Wu, Y., Vadgama, J.V. STAT3 activation in HER2overexpressing breast cancer promotes epithelial-mesenchymal transition and cancer stem cell traits. Int J Oncol. 44,403-11(2014).

34. Moore, R.G., Hill, E.K., Horan, T., Yano, N., Kim, K., MacLaughlan, S., LambertMesserlian, G., Tseng, Y.D., Padbury, J.F., Miller, M.C., Lange, T.S., Singh, R.K. Sci Rep. 4,3574(2014)

35. Bolger, A.M., Lohse, M., Usadel, B. Trimmomatic: a flexible trimmer for Illumina sequence data. Bioinformatics. 30,2114-2120(2014).

36. Dobin, A., Davis, C.A., Schlesinger, F., Drenkow, J., Zaleski, C., Jha, S., Batut, P., Chaisson, M., Gingeras, T.R. STAR: ultrafast universal RNA-seq aligner. Bioinformatics. 29,15-21(2012). 
37. R Core Team. R: A language and environment for statistical computing. R Foundation for Statistical Computing, Vienna, Austria. (2016). URL https://www.R-project.org/

38. Love, M.I., Huber, W., Anders, S. "Moderated estimation of fold change and dispersion for RNA-seq data with DESeq2." Genome Biology 15,550(2014).

39. Liao, Y., Smyth, GK., Shi, W. featureCounts: an efficient general-purpose program for assigning sequence reads to genomic features. Bioinformatics 30, 923-930(2014).

40. Heasley, L.R., Garcia III G., McMurray MA. Off-Target Effects of the Septin Drug Forchlorfenuron on Nonplant Eukaryotes. Eukaryotic Cell 13;1411-1420 (2014).

41. Slamon, D.J., Clark, G.M., Wong, S.G., Levin, W.J., Ullrich, A., McGuire, W.L. Human breast cancer: correlation of relapse and survival with amplification of the HER-2/neu oncogene. Science 235:177-182 (1987).

42. Pohlmann, P.R., Mayer, I.A., Mernaugh, R. Resistance to Trastuzumab in Breast Cancer. Clin Cancer Res.15:7479-7491 (2009).

43. Hayes, D.F., Yamauchi, H., Broadwater, G., Cirrincione, C.T., Rodrigue, S.P., Berry, D.A., Younger, J., Panasci, L.L., Millard, F., Duggan, D.B., Norton, L., Henderson, I.C.; Cancer and Leukemia Group B. Circulating HER-2/erbB-2/c-neu (HER-2) extracellular domain as a prognostic factor in patients with metastatic breast cancer: Cancer and Leukemia Group B Study 8662. Clin. Cancer Res.7,2703-11 (2001).

44. Belkacemi, Y., Hanna, N.E., Besnard, C., Majdoul, S., Gligorov, J. Local and Regional Breast Cancer Recurrences: Salvage Therapy Options in the New Era of Molecular Subtypes. Front Oncol. 8,112(2018).

45. Clayton, A.J., Danson, S., Jolly, S., Ryder, W.D., Burt, P.A., Stewart, A.L., Wilkinson, P.M., Welch, R.S., Magee, B., Wilson, G., Howell, A., Wardley, A.M. Incidence of cerebral metastases in patients treated with trastuzumab for metastatic breast cancer. $\mathrm{Br}$ J Cancer 91,639-643 (2004).

46. Morris PG, McArthur HL, Hudis CA. Therapeutic options for metastatic breast cancer. Expert Opin Pharmacother. 10,967-981(2009).

Author contributions: RS conceived the idea, designed study, synthesized compounds including UR214-9 and conducted animal experiments in team with LL. LL ran xenograft animal studies, recorded tumor size measurements and animal weights without involvements of RS. LR and JK participated in animal studies. CL conducted the docking studies. AJ, NK, PS, RP, AA and KKK conducted western blot, immunoprecipitation, cell viability and other supplementary studies. $\mathrm{VH}$ performed confocal microscopic studies. TC conducted antibody array, cell cycle and livedead cell assay experiments. RNA-seq data was generated and analyzed by the team of CB, JRM, EZ and JA. RS assembled the manuscript. RT, MTM, DL, RGM and SG reviewed the data and edited the manuscript. Every author read and edited the manuscript, and approved the present version of manuscript for submission.

Acknowledgements: KKK and RS are grateful to UR Ventures of University of Rochester for a pilot grant award. RS gratefully acknowledges Mae Goode Foundation award to partially support these studies.

Conflict of Interest: KKK, RBRT, RGM and RS are listed as the co-inventors on a provisional US patent application US62/894,424 covering UR214-9 and its analogs for the treatment of malignancies and other disease states orchestrated by septins. 